\title{
The periodic standing-wave approximation: eigenspectral computations for linear gravity and nonlinear toy models
}

\author{
Christopher Beetle \\ Department of Physics, Florida Atlantic University, Boca Raton, Florida 33431 \\ Benjamin Bromley \\ Department of Physics, University of Utah, Salt Lake City, Utah 84112 \\ Richard H. Price \\ Department of Physics $\&$ Astronomy and Center for Gravitational Wave Astronomy, \\ University of Texas at Brownsville, Brownsville, TX 78520
}

\begin{abstract}
The periodic standing wave approach to binary inspiral assumes rigid rotation of gravitational fields and hence helically symmetric solutions. To exploit the symmetry, numerical computations must solve for "helical scalars," fields that are functions only of corotating coordinates, the labels on the helical Killing trajectories. Here we present the formalism for describing linearized general relativity in terms of helical scalars and we present solutions to the mixed partial differential equations of the linearized gravity problem (and to a toy nonlinear problem) using the adapted coordinates and numerical techniques previously developed for scalar periodic standing wave computations. We argue that the formalism developed may suffice for periodic standing wave computations for post-Minkowskian computations and for full general relativity.
\end{abstract}

\section{INTRODUCTION}

The computational study of the inspiral of binary black holes is important for the understanding of gravitational wave signals, and is of inherent interest as a question in general relativity that can be answered only with computation. It has therefore become the focus of supercomputer codes that evolve Einstein's field equations forward in time from initial conditions chosen to represent a starting configuration of the inspiralling objects. The evolution codes, however, typically become unstable on a timescale (set by the size of the hole) short compared to a full orbit. Reliable calculations of the final plunge are now feasible 1, 2], the merger and ringdown of the final black hole fate of the system are handled well with perturbation theory [3] , and the early inspiral is well approximated with post-Newtonian computations [4]. What cannot be handled well is the intermediate phase of the inspiral, the late epoch during which nonlinear effects are too strong for a post-Newtonian approximation, but for which too many orbits remain for stable numerical evolution.

It has long been recognized that the basis of an approximation scheme should be the slow rate of inspiral, the small ratio of the orbital time to the radiation damping time [5, [6]. Through an adiabatic treatment of the slow inspiral, such an approximation could give answers about the radiation and rate of inspiral in the intermediate epoch. In addition, when the rate of inspiral becomes too rapid, the intermediate approximation could hand the problem off to numerical evolution codes to do the final orbit or two and the plunge. Along with the problem being handed off, would be the ideal initial data for the subsequent evolution. The need for and the concept of an intermediate approximation have been clear, but such an approximation has not been easy to implement. Along with several others [7, 8, 9, 10] we have based an approximation for slow inspiral on a numerical computation of no inspiral. That is, we seek a numerical solution of Einstein's equations for binary objects that are in circular periodic motion, and whose "helically symmetric" fields rotate rigidly with the source objects. (For a definition of helical symmetry, see Sec. II of Ref. 11].)

The universality of gravitation suggests that the unchanging motion of such a system is not compatible with outgoing radiation, and this intuitive suggestion is confirmed by the mathematics of the theory. We therefore seek a helically symmetric solution for the sources coupled to standing waves, not to outgoing waves. In a linear theory, standing waves, in the sense that we use the term, are a superposition of half-ingoing and half-outgoing solutions. From the fact that the solution, in linear theory, is half the superposition of the ingoing and outgoing solutions, one could extract the outgoing solution. The crux of our periodic standing wave (PSW) method is that even for highly nonlinear binary inspiral fields there is an "effective linearity." The standing wave solution, to good accuracy, is half the sum of the outgoing plus ingoing solutions despite the nonlinearities. In general relativity, therefore, our goal is to solve the standing wave numerical problem and from that solution to extract an approximation to the outgoing solution.

It is important to understand why effective linearity can be correct for inspiral. In the strong-field regions very 
close to the sources, the solution is very insensitive to the choice of distant radiative boundary conditions (ingoing, outgoing, standing wave). In this near-source region a superposition of half the ingoing and half the outgoing solution gives a good approximation solution, because it amounts to averaging two samples of the same thing. In the wave zone where the outgoing and the ingoing solutions are very different, the fields are weak enough that nonlinear effects are negligible, and once again we can superpose. The strong-field region and the boundary-influenced region should be widely separated unless the sources are rotating very close to $c$, in which case the wave zone will start just outside the sources. It is, however, not expected that ultrarelativistic source motion can occur during the slow inspiral epoch of motion.

We have recently 12 been able to confirm effective linearity. This confirmation has been achieved with a model problem, since the validity of effective linearity can only be carried out in a model problem. In general relativity, there can be no "true outgoing" solution available for confirmation until numerical evolution codes are fully developed. In addition, the numerical features of the helically symmetric standing wave calculation pose new challenges very different from those of evolution codes, and are best resolved in the simplest context possible.

The model problem in Ref. 12] used a nonlinear scalar field theory with a pair of diametrically opposite point-like scalar charges. The imposition of helical symmetry on the problem leads to a boundary value problem for a system of mixed (hyperbolic and elliptical) partial differential equations. To solve that boundary value problem efficiently, a set of numerical techniques was developed that we called the "eigenspectral" method. In the present paper we report two important steps toward using the PSW method for full general relativity: First, we develop the infrastructure for describing gravitational fields. In previous work with scalar toy models helical symmetry was imposed by simply requiring that the scalar field is a function only of three corotating coordinates (labels on the Killing trajectories). We find it useful to use the expression "helical scalars" for such functions that depend only on corotating coordinates. Our computation is done on a grid of corotating coordinates, so straightforward computations can only be carried out for helical scalars. But complications arise with tensor fields. The components of a helically symmetric tensor field are generally not helical scalars. A resolution of this difficulty is to compute only with projections of the tensor on a helically symmetric basis, that is, on a basis that is Lie dragged by the helical symmetry. These projections would be helical scalars. A helically symmetric basis, however, has its own dynamics, and complicates the time dependence of the projected fields. In the infrastructure developed here we show that the use of a corotating "pure-spin" basis leads to a remarkably simple description of helically symmetric tensorial fields. That infrastructure is presented in the explicit context of linearized general relativity.

The second step taken in this paper is to present numerical results showing that no new computational problems are encountered in dealing with the helical scalars of the linearized gravity problem. Since no new problems were anticipated this numerical work simply constitutes a confirmation that the "eigenspectral method," the set of techniques developed for scalar models, appear to work equally well for linearized gravity. Those techniques include the use of (i) "adapted coordinates," a corotating coordinate system that conforms well to the source regions and to the radiation field, (ii) "multipole filtering," the elimination of numerical noise associated with angular differencing, by keeping only a few multipoles of the adapted coordinates, and (iii) the modification of the multipoles so that they are computationally orthogonal at the level of machine precision. In this paper we provide numerical solutions using this set of techniques. Since these solutions differ very little from the numerical problems studied in detail in Ref. [12] the presentation of linearized results is brief. The solutions to nonlinear problems are much more difficult than those for linearized problems. Newton-Raphson iteration must be used, and convergence of the iterative process has been the major challenge in numerical work. Again, there is no apparent reason the problem should be more difficult for the tensor-based helical scalars than for the nonlinear scalar models of Ref. 12]. Nevertheless, it is an issue worth checking, and initial results are briefly reported showing that the scalar techniques work well for a model tensorial problem with a simple toy nonlinearity.

The rest of this paper is organized as follows. Section $\amalg$ gives a brief review of the scalar PSW problem in order to introduce adapted coordinates and the application of multipole filtering and the eigenspectral method in those coordinates. Section 11 presents the description of the helically symmetric fields of linearized gravity that is suitable for computation. The field equations are given for general corotating coordinates and series solutions are given, in corotating spherical coordinates, for the problem of two equal masses in circular orbits around each other. The field equations for linearized gravity using adapted coordinates are given in Sec.IV] In that section, also, are given the forms in adapted coordinates of other elements of the computational problem, the inner boundary condtions specifying the sources, and the outer boundary conditions specifying the nature of radiation. Section $\mathbf{\nabla}$ presents numerical results, comparing the series solutions of Sec. III with the solutions of the field equations using the eigenspectral method. In addition, in this section a description is given of a toy nonlinear modification to linearized gravity, and results are given demonstrating that the resulting "theory" can be solved with the techniques that worked for the scalar case. The implications for the next steps in the PSW program are discussed in Sec. VI] In particular, it is argued that almost all the infrastructure for solving the PSW problems in the post-Minkowski approximation and in full general relativity problem is probably established in the work with the linearized problem. The Appendix gives some detailed 
expressions needed for computations in adapted coordinates.

Throughout the paper we use units in which $c=G=1$ and we follow the conventions of Misner, Thorne and Wheeler 13.

\section{SCALAR MODELS, COORDINATES, AND NUMERICS}

\section{A. Nonlinear scalar models}

Here we review the model problem and numerical techniques of Ref. [12]. Our model problem was a nonlinear scalar field coupled to point-like sources in Minkowski space, and satisfying the field equation

$$
\Psi_{; \alpha ; \beta} g^{\alpha \beta}+F=\nabla^{2} \Psi-\frac{1}{c^{2}} \partial_{t}^{2} \Psi+F=\text { Source. }
$$

In principle, the source was taken to be two points of unit scalar charge in orbit around each other at angular frequency $\Omega$, and at radius $a$. In practice, the computational problem used inner boundary conditions on small, approximately spherical surfaces to represent the effect of a point source; no source term was included in the field equation that was computationally solved. The velocity parameter for the system $v=a \Omega$ was taken to be of order unity, representing the strong-field tight binary for which post-Newtonian approximations are inadequate.

The term $F$ contains the nonlinearity in our scalar model theory, and we found the following form, with parameters $\lambda$ and $\Psi_{0}$, to be useful:

$$
F=\frac{\lambda}{a^{2}} \frac{\Psi^{5}}{\Psi_{0}^{4}+\Psi^{4}}
$$

A crucial feature of $F$ is that, like the nonlinearities of general relativity, it is very large near the sources, and becomes negligible far from the sources. The $\lambda$ multiplier allowed us to vary the strength of the nonlinear term, and the $\Psi_{0}$ parameter allowed us to vary the profile of the nonlinearity in the strong field region.

Our scalar problem was defined by Eqs. (1) and (2), and by the source motion at angular frequency $\Omega$ in the equatorial plane. With standard spherical coordinates, helical symmetry can be imposed on the solution $\Psi(t, r, \theta, \phi)$ by restricting to solutions of the form $\Psi(r, \theta, \varphi)$, where $\varphi$ is the comoving azimuthal coordinate $\phi-\Omega t$. By restricting the solution in this way, we have eliminated the possibility of "evolution." For such helically symmetric solutions a change in time by $\Delta t$ is the same as a change the azimuthal angle $\Delta \phi=-\Omega \Delta t$.

When the restriction to helical symmetry is made, the field equation becomes

$$
\begin{aligned}
\mathcal{L} \Psi \equiv \frac{1}{r^{2}} \frac{\partial}{\partial r}( & \left.r^{2} \frac{\partial \Psi}{\partial r}\right)+\frac{1}{r^{2} \sin \theta} \frac{\partial}{\partial \theta}\left(\sin \theta \frac{\partial \Psi}{\partial \theta}\right) \\
& +\left[\frac{1}{r^{2} \sin ^{2} \theta}-\Omega^{2}\right] \frac{\partial^{2} \Psi}{\partial \varphi^{2}}=\text { Source }-F(\Psi) \equiv \sigma(\Psi),
\end{aligned}
$$

and the mixed nature of the partial differential equation becomes obvious. The principal part of this quasilinear equation is elliptic inside a cylinder at $r \sin \theta=1 / \Omega$, and hyperbolic outside that cylinder. The problem is to be solved with radiative conditions (ingoing, outgoing, or standing wave as described below) on a spherical surface at large distances from the sources. Well posed problems in physics typically supply cauchy data on open surfaces to hyperbolic equations, and Dirichlet or Neumann data on closed surfaces to elliptic equations. Our model is unusual in that it leads to a boundary value problem with "radiation" conditions on a closed surface surrounding a mixed problem. Though unusual, our problem is intuitively well posed, and passes a computational test: we have found no fundamental difficulty in solving models of this type numerically. Furthermore, a careful analysis 14 proves that solutions exist and are stable for a closely related problem.

"Standing wave" solutions - half ingoing and half outgoing - are at the heart of our method, but there is not an unambiguous definition of standing wave solutions in a nonlinear theory. Our procedure is to find the outgoing $\mathcal{L}_{\text {out }}^{-1}$ and ingoing $\mathcal{L}_{\text {in }}^{-1}$ Green functions for Eq. (3). In principle, we can then iterate to find a solution of Eq. (3). The iteration

$$
\Psi_{\text {out }}^{(n+1)}=\mathcal{L}_{\text {out }}^{-1}\left[\sigma\left(\Psi_{\text {out }}^{(n)}\right)\right],
$$

if it converges, gives $\Psi_{\text {out }}$, our nonlinear outgoing solution (and similarly for $\Psi_{\text {in }}$ ), while the convergent result of

$$
\Psi_{\text {std }}^{(n+1)}=\frac{1}{2}\left(\mathcal{L}_{\text {out }}^{-1}+\mathcal{L}_{\text {in }}^{-1}\right)\left[\sigma\left(\Psi_{\text {std }}^{(n)}\right)\right]
$$


is what we mean by our nonlinear standing wave solution $\Psi_{\text {std }}$. The standing wave solution $\Psi_{\text {std }}$ is fundamentally different from $\left(\Psi_{\text {out }}+\Psi_{\text {in }}\right) / 2$, but if effective linearity is correct, the two are very nearly equal. (Note: In practice, for strong nonlinearities, the direct iteration described above must be replaced by Newton-Raphson iteration.)

A central idea of the PSW approximation is that the "exact" (i.e., numerical) solution to the standing wave problem is an excellent approximation to half the sum of the outgoing and ingoing solutions. If this is so, it means that from the standing wave solution we should be able to extract an excellent approximation to the outgoing and the ingoing solutions. (It should be noted that this statement is meaningful for nonlinear model field theories in which there is meaning to an outgoing helically symmetric or ingoing helically symmetric solution to the field theory. For general relativity, a helically symmetric spacetime with outgoing radiation is impossible. As explained in Ref. [12], the relevance and justification of the PSW approximation for full general relativity lie in the fact that the method gives an approximation only for a limited region of spacetime.)

The extraction of the approximate nonlinear outgoing solution from the computed standing wave solution is a direct application of the concepts underlying the argument for effective linearity. In the weak wave zone, far from the sources, the field theory is very nearly linear and the solution must be very nearly a standing wave solution to that linear theory. It is, therefore, straightforward to deconstruct it into outgoing and ingoing solutions. The extracted outgoing solution can be continued inward through the induction zone into the near-field zone. If the theory were completely linear, this continuation would be valid up to the source points. Due to the nonlinearity, however, this procedure is no longer valid at distances so close to the source points that the nonlinearity of the theory is important.

In the near field region close to the source points, at a distance small compared to a wavelength, the solution is highly insensitive to the nature of the boundary conditions (i.e., whether they are outgoing or ingoing). Here, we can use the standing wave solution itself as the outgoing solution. This near field solution should extend out to the weak field region and overlap with the extracted outgoing solution described above. These two solutions, the weak field outgoing solution outside the strong field region, and the standing wave solution in the strong field region, are then patched together, with some blending in the region of overlap, and the result is the PSW extracted approximation to the nonlinear outgoing solution.

\section{B. Adapted coordinates}

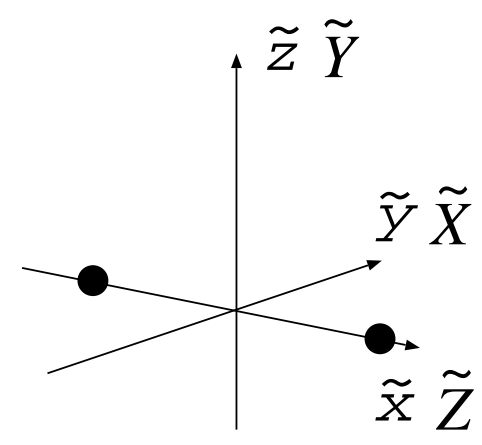

FIG. 1: Two systems of corotating Cartesian coordinates.

It is useful to identify a number of coordinate systems to describe the physical problem, including three Cartesian systems. The system $\{x, y, z\}$ is that of inertial Cartesian system in which the $z$ axis is the rotation axis for the source objects, with rotation in the positive sense about the $z$ axis. In general we use tildes to distinguish the corotating version of a quantity when that quantity occurs in forms both related to inertial and to corotating systems. The set $\{\widetilde{x}, \widetilde{y}, \widetilde{z}\}$, in this sense, is the corotating set of Cartesian coordinates for which $\widetilde{z}=z$ and for which the source points remain fixed on the $\widetilde{x}$ axis. The system $\{r, \theta, \phi\}$ is that of inertial spherical coordinates defined in the usual way relative to $\{x, y, z\}$. The system $\{r, \theta, \varphi\}$ is a set of coroting spherical polar coordinates, defined by the usual transformation relative to $\{\widetilde{x}, \widetilde{y}, \widetilde{z}\}$. The two systems of spherical coordinates are related by $\varphi=\phi-\Omega t$. The Cartesian system $\{\widetilde{X}, \widetilde{Y}, \widetilde{Z}\}$ is a convenient renaming of $\{\widetilde{x}, \widetilde{y}, \widetilde{z}\}$, with $\widetilde{Y}$ the rotational axis, and $\widetilde{Z}$, the axis through the source points.

Our adapted coordinate system $\{\chi, \Theta, \Phi\}$ is a corotating two-center bipolar coordinate system defined, relative to 
the $\{\tilde{X}, \widetilde{Y}, \widetilde{Z}\}$ system, by

$$
\begin{aligned}
\chi & \equiv\left\{\left[(\widetilde{Z}-a)^{2}+\widetilde{X}^{2}+\widetilde{Y}^{2}\right]\left[(\widetilde{Z}+a)^{2}+\widetilde{X}^{2}+\widetilde{Y}^{2}\right]\right\}^{1 / 4} \\
\Theta & \equiv \frac{1}{2} \tan ^{-1}\left(\frac{2 \widetilde{Z} \sqrt{\widetilde{X}^{2}+\widetilde{Y}^{2}}}{\widetilde{Z}^{2}-a^{2}-\widetilde{X}^{2}-\widetilde{Y}^{2}}\right) \\
\Phi & \equiv \tan ^{-1}(\widetilde{Y} / \widetilde{X}),
\end{aligned}
$$

where $a$ is the distance from the center to each of the source points.

For $\chi \gg a$, the adapted coordinates become a corotating system of spherical coordinates defined relative to $\{\widetilde{X}, \widetilde{Y}, \widetilde{Z}\}$. (That is, the $\widetilde{Z}$ axis is the azimuthal axis for the $\Phi$ coordinate.) The adapted coordinates, pictured in Fig. 2] are discussed in greater detail in Ref. [12].
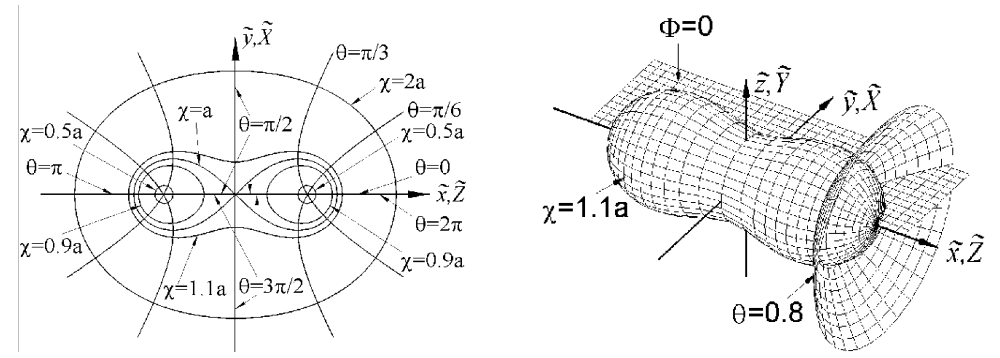

FIG. 2: Two-center bipolar adapted coordinates. On the left is shown curves of coordinates $\chi$ and $\Theta$ in the $\Phi=0$ orbital plane. The surfaces on the right show surfaces of constant $\chi, \Theta$, and $\Phi$. Here $a$ is the distance from either of the two centers (locations of the point sources) to the midpoint between the centers. The $\widetilde{x}, \widetilde{y}, \widetilde{z}$ coordinate system is a corotating Cartesian system for which the $\widetilde{z}$ axis is the rotation axis; for the $\widetilde{X}, \widetilde{Y}, \widetilde{Z}$ a corotating Cartesian system the $\widetilde{Z}$ axis is the line through the centers.

We originally [10] solved the model problem of Eq. (3) in the $\{r, \theta, \varphi\}$ system with more-or-less straightforward finite differencing and direct matrix inversion. (The mixed nature of the partial differential equations prevents the use of such efficient techniques as overrelaxaton.) This approach was successful (iterations converged) for models with a limited range of source velocities and nonlinearities. Subsequently we developed an innovative numerical method that gave remarkably good results for the scalar problem, with very little computational cost. The new method is based on three elements.

First, we used the adapted coordinates discussed above. The solutions to the finite difference form of Eq. (1) in these coordinates turned out to be plagued by what appeared to be angular noise. This noise was eliminated with multipole filering, a form of smoothing of the angular variation. In this method the scalar field was expanded in spherical harmonics of the angular functions $\Theta$ and $\Phi$. The fact that the adapted coordinates are well suited both to the source structure and to the radiation field suggests that good accuracy can be achieved when only a few multipoles are kept. In fact, good accuracy was found when only the monopole and quadrupole were kept in the case of sources speeds around $0.3 c-0.4 c$ or less. At higher speeds the radiation field develops sharper gradients and more multipoles must be kept to achieve reasonable accuracy.

\section{C. inner and outer boundary conditions}

For identical point sources of unit scalar charge moving in the equatorial plane at angular velocity $\Omega$ in circular orbits of radius $a$, the source used in Eq. (11) was

$$
S=-\gamma^{-1} \frac{\delta(r-a)}{a^{2}} \delta(\theta-\pi / 2)[\delta(\varphi)+\delta(\varphi-\pi)],
$$

with $\gamma \equiv 1 / \sqrt{1-v^{2}}=1 / \sqrt{1-a^{2} \Omega^{2}}$. For this source, it was shown in Ref. 12] that the small- $\chi$ limit of $\Phi$ is

$$
\Psi=\frac{1}{4 \pi} \frac{2 a}{\chi^{2}} \frac{1}{\sqrt{1+\gamma^{2} v^{2} \sin ^{2} 2 \Theta \cos ^{2} \Phi}} .
$$


We take this as an inner boundary condition at a small value of $\chi$ that determines one limit of our computational grid.

The outer boundary condition used is simply the ingoing or outgoing Sommerfeld condition expressed in terms of the adapted coordinates. In Ref. [12] it was shown that in adapted coordinates this condition becomes

$$
\frac{1}{\chi} \frac{\partial}{\partial \chi}(\chi \Psi)= \pm \Omega\left(\cos \Phi \frac{\partial \Psi}{\partial \Theta}-\frac{\cos \Theta}{\sin \Theta} \sin \Phi \frac{\partial \Psi}{\partial \Phi}\right)
$$

in which the upper and lower signs correspond, respectively, to the outgoing and ingoing conditions.

\section{D. multipole filtering and the eigenspectral technique}

In practice, multipole filtering was carried out as follows. If there are $N$ grid locations $\Theta_{i}$, $\Phi_{j}$, of the angular coordinates $\Theta, \Phi$, then at each value of the radial coordinate $\chi$ there are $N$ values of the scalar field $\Psi\left(\chi, \Theta_{i}, \Phi_{j}\right)$. In multipole filtering, a set of $M \leq N$ spherical harmonics $Y_{i j}$ is used, at each value of $\chi$, as weighting factors for these scalar field values, and $M$ weighted sums (i.e., multipole projections) are taken of the scalar field values. In the same manner, the $N$ field equations at $\chi$ are projected into $M$ sums. Those $M$ projected equations are then solved for the $M$ projections of the field.

It was found that this procedure did not work if the multipole weights $Y_{i j}$ were simply taken to be $Y_{L m}\left(\Theta_{i}, \Phi_{j}\right)$, the continuum spherical harmonics evaluated at the discrete angular grid locations. These projection weights are only orthogonal in the continuum limit, and their failure to be numerically orthogonal to high precision was the probable source of angular noise that plagued computations. Slightly modified weights, in place of the grid-evaluated spherical harmonics, gave us weights that was orthogonal to the level of machine precision. The use of these modified weights in multipole filtering eliminated the problem of angular noise, while at the same time significantly reducing the number of equations to be solved, and hence reducing the computational burden. See Ref. 12] for details.

We focused, in Ref. [12], on the most important question that can be answered with these models and numerical methods: Does effective linearity work? Can we extract a good approximation to the outgoing nonlinear problem from the sort of standing wave computation we will be limited to when dealing with Einstein's theory? Figure 3 gives strong evidence that we can. In that figure, the computed outgoing nonlinear solution is shown as a solid curve. The data-type points represent the outgoing solution extracted in the manner described above in Sec. 【A For the parameters $\lambda=-15$ and $\Psi_{0}=0.15$ in Fig. [3 nonlinearities are significant, strong enough to reduce field strength by around two-thirds. The outgoing and standing wave solutions were each computed by the Newton-Raphson version of the iteration in Eqs. (4),(5). We have run models with much stronger nonlinearity and have found equally good agreement of the true outgoing solution and the extracted approximation. The validity of effective linearity should, in fact, become questionable not for stronger nonlinearity, but only for physically implausible high source velocity.

In addition to confirming effective linearity, computation with the model has also allowed some early insights about sensitivity to source details. By varying the multipole content of the inner boundary data we explored the impact of source structure on the radiation field. The result (detailed in Ref. [12]) is in perfect accord with physical intuition; the radiation is insensitive to source structure unless the source size becomes comparable to the separation of the sources (i.e., unless the moments ascribable to the structure of the individual sources are comparable to the quadrupole moment due to the separation of the mass points). The equivalent question for Einstein's theory is more difficult, but we should eventually be able to give clear quantitative answers.

\section{THE DESCRIPTION OF HELICALLY SYMMETRIC LINEARIZED GRAVITATIONAL FIELDS}

\section{A. The physical problem}

We use the standard description and notation for the linearization of Einstein's field equations (see Chap. 8 of Ref. [13]). The perturbations from flat spacetime $h_{\mu \nu}$ are defined by

$$
g_{\mu \nu}=\eta_{\mu \nu}+h_{\mu \nu}
$$

and the trace-reversed perturbations $\bar{h}_{\mu \nu}$ are defined by

$$
\bar{h}_{\mu \nu} \equiv h_{\mu \nu}-\frac{1}{2} \eta_{\mu \nu} h
$$



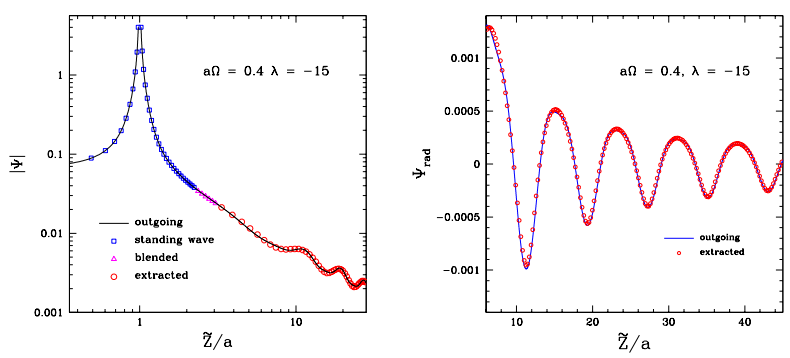

FIG. 3: The computed nonlinear outgoing solution compared with an approximate outgoing solution extracted from the computed nonlinear standing wave solutions. The solid curves show the computed outgoing solution for a scalar model with nonlinearity parameters $\lambda=-15$ and $\Psi_{0}=-0.15$ and with source velocity $a \Omega=0.4 c$. The data-like markers show the approximate outgoing solution extracted from the standing wave solution; the shape of the markers indicate whether the extracted solution corresponds to the deconstruction of the linearized solution, the standing wave solution, or a region of blending of the linearized outgoing and standing wave solutions. The computations of both the standing wave solution and the outgoing solution were carried out using quadrant symmetry with a $40 \times 80$ angular grid, and with 16001 divisions in $\chi$ ranging from $\chi=0.02 a$ to $80 a$. The multipole filtering kept only the multipoles corresponding to $\ell=0,2$ and 4 .

where $h \equiv \eta^{\mu \nu} h_{\mu \nu}$. For linearized computations, $\bar{h}_{\mu \nu}$ is treated as a tensorial field in Minkowski spacetime, and indices are raised and lowered with the Minkowskian metric $\eta_{\mu \nu}$. In the gravitational Lorentz gauge

$$
\bar{h}_{, \mu}^{\mu \nu}=0,
$$

the linearized field equations of general relativity are

$$
\bar{h}_{\mu \nu, \alpha}^{\alpha}=-16 \pi T_{\mu \nu} .
$$

Our physical problem is that of two points, each of mass $m_{0}$, in circular orbits, with radius $a$, angular velocity $\Omega$, and hence speed $v=a \Omega$ through the background Minkowski spacetime. The general form of the stress-energy source for linearized theory 15$]$ is

$$
T^{\mu \nu}=m_{0} \int_{-\infty}^{\infty} \delta^{(4)}\left(x^{\alpha}-z^{\alpha}(\tau)\right) \frac{d z^{\mu}}{d \tau} \frac{d z^{\nu}}{d \tau} d \tau=m_{0} \frac{U^{\mu} U^{\nu}}{U^{0}} \frac{\delta(r-R(t))}{r^{2}} \delta^{(2)}(\Omega-\Omega(t)),
$$

in which

$$
U^{0} \equiv \gamma=1 / \sqrt{1-v^{2}} \quad U^{x}=\mp v \gamma \sin \Omega t \quad U^{y}= \pm v \gamma \cos \Omega t .
$$

The signs of $U^{x}$ and $U^{y}$ are different for the two source particles. One, call it particle 1 , is at $\phi=\Omega t$; the other, particle 2, is at $\phi=\Omega t+\pi$. In Eq. (17) the upper sign corresponds to particle 1, the lower to particle 2. The explicit nonvanishing components, in the inertial $t, r, \theta, \phi$ system, are then,

$$
\begin{gathered}
T^{t t}=m_{0} \gamma \frac{\delta(r-a)}{a^{2}} \delta(\theta-\pi / 2)[\delta(\varphi)+\delta(\varphi-\pi)] \\
T^{t x}=\mp v \sin \Omega t T^{t t} \quad T^{t y}= \pm v \cos \Omega t T^{t t} \\
T^{x x}=v^{2} \sin ^{2} \Omega t T^{t t} \quad T^{y y}=v^{2} \cos ^{2} \Omega t T^{t t} \quad T^{x y}=-v^{2} \sin \Omega t \cos \Omega t T^{t t} .
\end{gathered}
$$

We use this helically symmetric stress energy as an explicit source when we derive the series solutions to Eq. (15). For the solution of Eq. (15) via the eigenpectral method of Ref. [12], however, we find $\bar{h}_{\alpha \beta}$ in the limit of small distance from the particles, and impose this solution as inner boundary conditions on the homogeneous form of Eq. (15). To get the near-particle solution we use the Liénard-Wiechert solution of Eq. (15)

$$
\bar{h}^{\alpha \beta}(\vec{x})=-\left.4 m_{0} \frac{U^{\alpha}(\tau) U^{\beta}(\tau)}{\vec{U} \cdot(\vec{x}-\vec{r}(\tau))}\right|_{\text {ret }} .
$$


The retardation condition "ret," means that $\tau$ is to be evaluated at time such that

$$
|\vec{x}-\vec{r}(\tau)|=0 .
$$

For the particle at $\varphi=0$, we now evaluate this approximately by assuming that the particle moves with constant velocity $x=a, y=a \Omega t_{\text {part }}=v t_{\text {part }}, z=0$, through the inertial coordinate frame. At field point $t, x, y, z$ we have the following retardation condition for the particle at $x=a$ :

$$
0=-\left(t-t_{\text {part }}\right)^{2}+(x-a)^{2}+\left(y-v t_{\text {part }}\right)^{2}+z^{2},
$$

with the solution

$$
t_{\mathrm{part}}=\gamma^{2}\left[t-y v-\sqrt{(t-v y)^{2}+\left(r^{2}-t^{2}\right) / \gamma^{2}}\right]
$$

in which

$$
r^{2} \equiv(x-a)^{2}+z^{2}+y^{2} .
$$

To express this in corotating coordinates, we next introduce the notations and approximations

$$
y=\widetilde{y}+v t \quad \widetilde{x}=x \quad \widetilde{z}=z \quad \widetilde{r}^{2} \equiv(\widetilde{x}-a)^{2}+\widetilde{y}^{2}+\widetilde{z}^{2} .
$$

With these, Eq. (24) simplifies to

$$
t_{\mathrm{part}}=t-v \gamma^{2} \widetilde{y}-\gamma \sqrt{\widetilde{r}^{2}+\gamma^{2} v^{2} \widetilde{y}^{2}}
$$

and, finally, we can evaluate

$$
\begin{gathered}
\left.\vec{U} \cdot(\vec{x}-\vec{r}(\tau))\right|_{\mathrm{ret}}=-U^{0}\left(t-t_{\mathrm{part}}\right)+U^{y}\left(y-v t_{\mathrm{part}}\right) \\
=-\gamma\left[t-t_{\mathrm{part}}-v\left(y-v t_{\mathrm{part}}\right)\right] \\
=-\gamma^{-1}\left(t-t_{\mathrm{part}}\right)+v \gamma \widetilde{y}=-\sqrt{\widetilde{r}^{2}+\gamma^{2} v^{2} \widetilde{y}^{2}} .
\end{gathered}
$$

With Eqs. (17) and (28), the expression in Eq. (21) gives us the inner boundary conditions to be used for solving Eq. (15):

$$
\begin{aligned}
\bar{h}^{n n} & =4 m_{0} \frac{\gamma^{2}}{\sqrt{\widetilde{r}^{2}+\gamma^{2} v^{2} \widetilde{y}^{2}}} \\
\bar{h}^{n x}=\bar{h}^{x n} & =4 m_{0} \frac{\gamma^{2}}{\sqrt{\widetilde{r}^{2}+\gamma^{2} v^{2} \widetilde{y}^{2}}}[\mp v \sin \Omega t] \\
\bar{h}^{n y}=\bar{h}^{y n} & =4 m_{0} \frac{\gamma^{2}}{\sqrt{\widetilde{r}^{2}+\gamma^{2} v^{2} \widetilde{y}^{2}}}[ \pm v \cos \Omega t] \\
\bar{h}^{x x} & =4 m_{0} \frac{\gamma^{2}}{\sqrt{\widetilde{r}^{2}+\gamma^{2} v^{2} \widetilde{y}^{2}}}\left[v^{2} \sin ^{2} \Omega t\right] \\
\bar{h}^{y y} & =4 m_{0} \frac{\gamma^{2}}{\sqrt{\widetilde{r}^{2}+\gamma^{2} v^{2} \widetilde{y}^{2}}}\left[v^{2} \cos ^{2} \Omega t\right] \\
\bar{h}^{x y}=\bar{h}^{y x} & =4 m_{0} \frac{\gamma^{2}}{\sqrt{\widetilde{r}^{2}+\gamma^{2} v^{2} \widetilde{y}^{2}}}\left[-v^{2} \cos \Omega t \sin \Omega t\right] .
\end{aligned}
$$

The outer boundary conditions, roughly speaking, are the conditions that nonradiative parts of the field fall off as $1 / r^{n}$, and the radiative parts of the field satisfy simple ingoing or outgoing Sommerfeld conditions. The details are given after the presentation of the formalism for describing helically symmetric tensorial fields. 


\section{B. Description of helically symmetric tensorial fields}

Imposing helical symmetry on the tensorial field means that the Lie derivative of $\bar{h}_{\mu \nu}$ vanishes along the helical Killing field, or

$$
\mathcal{L}_{\xi}\left(\bar{h}_{\mu \nu}\right)=0
$$

Here the helical Killing vector is

$$
\xi=\partial_{t}+\Omega \partial_{\phi}=\partial_{t}
$$

The first expression gives $\xi$ in terms of the inertial spherical background coordinates $t, r, \theta, \phi$, and the second in terms of the corotating spherical background coordinates $t, r, \theta, \varphi=\phi-\Omega t$. As explained in Sec. 【 our computational unknowns are fields on a grid of corotating coordinates. We must therefore cast the field equations in terms of a set of functions that are "helical scalars," functions only of the spatial corotating Minkowski coordinates such as $\{r, \theta, \varphi\}$ $\{\widetilde{x}, \widetilde{y}, \widetilde{x}\},\{\widetilde{X}, \widetilde{Y}, \widetilde{Z}\}$, or the adapted coordinates $\{\chi, \Theta, \Phi\}$.

The key to this is to project on a corotating basis. A corotating vector $\widetilde{\mathbf{V}}$ is one for which $\mathcal{L}_{\xi}(\widetilde{\mathbf{V}})=0$. If $\widetilde{\mathbf{V}}$ and $\widetilde{\mathbf{W}}$ are both corotating vectors, and $\bar{h}_{\mu \nu}$ is helically symmetric, then $h_{\mu \nu} V^{\mu} W^{\nu}$ is a helically symmetric scalar, a function only of three corotating coordinates. Our approach, then is to use a coroating basis and to project all components of $h_{\mu \nu}$ on this basis.

If not done with some care, projection on a corotating basis, can destroy the simplicity of the linearized field equations (15). Since the inertial basis is covariantly constant, the equations separate for the components of $\bar{h}_{\mu \nu}$; each component satisfies its own equation, and the system of equations separates into a set of scalar-like equations. This is not true in general for the scalars formed by projections with the corotating bases. Choices can be made, however, that result in a high degree of separation, and a very simple set of equations.

To achieve this simplicity we start by defining the covariantly constant orthonormal inertial basis system for the Minkowski background:

$$
\mathbf{n}=\partial_{t} \quad \mathbf{e}_{x}=\partial_{x} \quad \mathbf{e}_{y}=\partial_{y} \quad \mathbf{e}_{z}=\partial_{z} \quad .
$$

Here we closely follow the analysis given by Thorne 16]. That analysis uses the method of Mathews [17], and introduces a set of second rank symmetric spatial basis tensors $\mathbf{t}_{2, j}$ of spin 2, i.e., tensors that transform among themselves as an irreducible represenation of the rotation group of order 2 . To these we add an additional spin 0 spatial tensor $\mathbf{t}_{0,0}$ and the spin 0 and spin 1 second-rank symmetric tensors needed to include the timelike direction

$$
\begin{aligned}
\mathbf{t}_{n n} & \equiv \mathbf{n n} \\
\mathbf{t}_{n 0} & \equiv \mathbf{n e}_{z} \\
\mathbf{t}_{n, \pm 1} & \equiv \frac{\mp 1}{2}\left[\mathbf{n}\left(\mathbf{e}_{x} \pm i \mathbf{e}_{y}\right)+\left(\mathbf{e}_{x} \pm i \mathbf{e}_{y}\right) \mathbf{n}\right] \\
\mathbf{t}_{0,0} & \equiv \frac{1}{\sqrt{3}}\left[\mathbf{e}_{x} \mathbf{e}_{x}+\mathbf{e}_{y} \mathbf{e}_{y}+\mathbf{e}_{z} \mathbf{e}_{z}\right] \\
\mathbf{t}_{2,0} & \equiv \frac{-1}{\sqrt{6}}\left[\mathbf{e}_{x} \mathbf{e}_{x}+\mathbf{e}_{y} \mathbf{e}_{y}-2 \mathbf{e}_{z} \mathbf{e}_{z}\right] \\
\mathbf{t}_{2, \pm 1} & \equiv \mp \frac{1}{2}\left[\mathbf{e}_{x} \mathbf{e}_{z}+\mathbf{e}_{z} \mathbf{e}_{x}\right]-\frac{1}{2} i\left[\mathbf{e}_{y} \mathbf{e}_{z}+\mathbf{e}_{z} \mathbf{e}_{y}\right] \\
\mathbf{t}_{2, \pm 2} & \equiv \frac{1}{2}\left[\mathbf{e}_{x} \mathbf{e}_{x}-\mathbf{e}_{y} \mathbf{e}_{y} \pm i\left(\mathbf{e}_{y} \mathbf{e}_{x}+\mathbf{e}_{x} \mathbf{e}_{y}\right)\right]
\end{aligned}
$$

We next define the corotating equivalents to the basis vectors

$$
\widetilde{\mathbf{n}}=\mathbf{n} \quad \widetilde{\mathbf{e}}_{x}=\mathbf{e}_{x} \cos \Omega t+\mathbf{e}_{y} \sin \Omega t \quad \widetilde{\mathbf{e}}_{x}=-\mathbf{e}_{x} \sin \Omega t+\mathbf{e}_{y} \cos \Omega t \quad \widetilde{\mathbf{e}}_{z}=\mathbf{e}_{z},
$$

and we use these to define the corotating equivalents of the basis tensors:

$$
\begin{aligned}
\widetilde{\mathbf{t}}_{n n} & \equiv \widetilde{\mathbf{n}} \widetilde{\mathbf{n}}=\mathbf{t}_{n n} \\
\widetilde{\mathbf{t}}_{n 0} & \equiv \widetilde{\mathbf{n}} \widetilde{\mathbf{e}}_{z}=\mathbf{t}_{n 0} \\
\widetilde{\mathbf{t}}_{n, \pm 1} & \equiv \frac{\mp 1}{2}\left[\widetilde{\mathbf{n}}\left(\widetilde{\mathbf{e}}_{x} \pm i \widetilde{\mathbf{e}}_{y}\right)+\left(\widetilde{\mathbf{e}}_{x} \pm i \widetilde{\mathbf{e}}_{y}\right) \widetilde{\mathbf{n}}\right]=e^{\mp i \Omega t} \mathbf{t}_{n, \pm 1} \\
\widetilde{\mathbf{t}}_{0,0} & \equiv \frac{1}{\sqrt{3}}\left[\widetilde{\mathbf{e}}_{x} \widetilde{\mathbf{e}}_{x}+\widetilde{\mathbf{e}}_{y} \widetilde{\mathbf{e}}_{y}+\widetilde{\mathbf{e}}_{z} \widetilde{\mathbf{e}}_{z}\right]=\mathbf{t}_{0,0} \\
\widetilde{\mathbf{t}}_{2,0} & \equiv \frac{-1}{\sqrt{6}}\left[\widetilde{\mathbf{e}}_{x} \widetilde{\mathbf{e}}_{x}+\widetilde{\mathbf{e}}_{y} \widetilde{\mathbf{e}}_{y}-2 \widetilde{\mathbf{e}}_{z} \widetilde{\mathbf{e}}_{z}\right]=\mathbf{t}_{2,0} \\
\widetilde{\mathbf{t}}_{2, \pm 1} & \left.\equiv \mp \frac{1}{2}\left[\widetilde{\mathbf{e}}_{x} \widetilde{\mathbf{e}}_{z}+\widetilde{\mathbf{e}}_{z} \widetilde{\mathbf{e}}_{x}\right]-\frac{1}{2} i \widetilde{\mathbf{e}}_{y} \widetilde{\mathbf{e}}_{z}+\widetilde{\mathbf{e}}_{z} \widetilde{\mathbf{e}}_{y}\right]=e^{\mp i \Omega t} \mathbf{t}_{2, \pm 1} \\
\widetilde{\mathbf{t}}_{2, \pm 2} & \equiv \frac{1}{2}\left[\widetilde{\mathbf{e}}_{x} \widetilde{\mathbf{e}}_{x}-\widetilde{\mathbf{e}}_{y} \widetilde{\mathbf{e}}_{y} \pm i\left(\widetilde{\mathbf{e}}_{y} \widetilde{\mathbf{e}}_{x}+\widetilde{\mathbf{e}}_{x} \widetilde{\mathbf{e}}_{y}\right)\right]=e^{\mp 2 i \Omega t} \mathbf{t}_{2, \pm 2} .
\end{aligned}
$$


Note that all the tensor bases are orthonormal in the following sense:

$$
\left(\mathbf{t}_{i, j}\right)^{*} \cdot\left(\mathbf{t}_{k, p}\right)=\left(\widetilde{\mathbf{t}}_{i, j}\right)^{*} \cdot\left(\widetilde{\mathbf{t}}_{k, p}\right)=\delta_{i k} \delta_{j p},
$$

and that the tensor $\bar{h}$ can be written as

$$
\begin{gathered}
\overline{\mathbf{h}}=\Psi^{(n n)} \mathbf{t}_{n n}+\Psi^{(n 0)} \mathbf{t}_{n 0}+\Psi^{(n 1)} \mathbf{t}_{n 1}+\Psi^{(n,-1)} \mathbf{t}_{n,-1}+\Psi^{(00)} \mathbf{t}_{00}+\Psi^{(20)} \mathbf{t}_{20}+ \\
\Psi^{(21)} \mathbf{t}_{21}+\Psi^{(2,-1)} \mathbf{t}_{2,-1}+\Psi^{(22)} \mathbf{t}_{22}+\Psi^{(2,-2)} \mathbf{t}_{2,-2}
\end{gathered}
$$

or as

$$
\begin{gathered}
\overline{\mathbf{h}}=\widetilde{\Psi}^{(n n)} \widetilde{\mathbf{t}}_{n n}+\widetilde{\Psi}^{(n 0)} \widetilde{\mathbf{t}}_{n 0}+\widetilde{\Psi}^{(n 1)} \widetilde{\mathbf{t}}_{n 10}+\widetilde{\Psi}^{(n,-10)} \widetilde{\mathbf{t}}_{n,-1}+\widetilde{\Psi}^{(00)} \widetilde{\mathbf{t}}_{00}+\widetilde{\Psi}^{(20)} \widetilde{\mathbf{t}}_{20}+ \\
\widetilde{\Psi}^{(21)} \widetilde{\mathbf{t}}_{21}+\widetilde{\Psi}^{(2,-1)} \widetilde{\mathbf{t}}_{2,-1}+\widetilde{\Psi}^{(22)} \widetilde{\mathbf{t}}_{22}+\widetilde{\Psi}^{(2,-2)} \widetilde{\mathbf{t}}_{2,-2} .
\end{gathered}
$$

From Eq. (53) we get

$$
\begin{aligned}
\Psi^{(n n)} & =\bar{h}^{t t} \\
\Psi^{(n 0)} & =\bar{h}^{t z} \\
\Psi^{(n 1)} & =-\bar{h}^{t x}+i \bar{h}^{t y} \\
\Psi^{(00)} & =\frac{1}{\sqrt{3}}\left[\bar{h}^{x x}+\bar{h}^{y y}+\bar{h}^{z z}\right] \\
\Psi^{(20)} & =\frac{-1}{\sqrt{6}}\left[\bar{h}^{x x}+\bar{h}^{y y}-2 \bar{h}^{z z}\right] \\
\Psi^{(21)} & =-\bar{h}^{x z}+i \bar{h}^{y z} \\
\Psi^{(22)} & =\frac{1}{2}\left[\bar{h}^{x x}-\bar{h}^{y y}\right]-i \bar{h}^{y x} .
\end{aligned}
$$

Here and below, we have dropped functions that are redundant due to the relations

$$
\Psi^{(n,-1)}=-\left(\Psi^{(n 1)}\right)^{*} \quad \Psi^{(2,-1)}=-\left(\Psi^{(21)}\right)^{*} \quad \Psi^{(2,-2)}=\left(\Psi^{(22)}\right)^{*} .
$$

These relations are also true if tildes are placed over all variables. From the rotation behaviors of the $\widetilde{\mathbf{t}}$ basis, given above, the rotation laws for the $\Psi$ fields are:

$$
\begin{aligned}
& \widetilde{\Psi}^{(n n)}=\Psi^{(n n)} \\
& \widetilde{\Psi}^{(n 0)}=\Psi^{(n 0)} \\
& \widetilde{\Psi}^{(n 1)}=e^{i \Omega t} \Psi^{(n 1)}=U^{(n 1)}+i V^{(n 1)} \\
& \widetilde{\Psi}^{(00)}=\Psi^{(00)} \\
& \widetilde{\Psi}^{(20)}=\Psi^{(20)} \\
& \widetilde{\Psi}^{(21)}=e^{i \Omega t} \Psi^{(21)}=U^{(21)}+i V^{(21)} \\
& \widetilde{\Psi}^{(22)}=e^{2 i \Omega t} \Psi^{(22)}=U^{(22)}+i V^{(22)},
\end{aligned}
$$

where $U^{(a b)}$ and $V^{(a b)}$ are real functions.

We define projections of the stress energy by analogy with the projections of the $\bar{h}$ perturbations,

$$
\begin{aligned}
& \mathcal{T}^{(n n)}=T^{t t}=\widetilde{\mathcal{T}}^{(n n)} \\
& \mathcal{T}^{(n 0)}=T^{t z}=\widetilde{\mathcal{T}}^{(n 0)} \\
& \mathcal{T}^{(n 1)}=-T^{t x}+i T^{t y}=e^{-i \Omega t} \widetilde{\mathcal{T}}^{(n 1)} \\
& \mathcal{T}^{(00)}=\frac{1}{\sqrt{3}}\left[T^{x x}+T^{y y}+T^{z z}\right]=\widetilde{\mathcal{T}}^{(00)} \\
& \mathcal{T}^{(20)}=\frac{-1}{\sqrt{6}}\left[T^{x x}+T^{y y}-2 T^{z z}\right]=\widetilde{\mathcal{T}}^{(20)} \\
& \mathcal{T}^{(21)}=-T^{x z}+i T^{y z}=e^{-i \Omega t} \widetilde{\mathcal{T}}^{(21)} \\
& \mathcal{T}^{(22)}=\frac{1}{2}\left[T^{x x}-T^{y y}\right]-i T^{y x}=e^{-2 i \Omega t} \widetilde{\mathcal{T}}^{(22)} .
\end{aligned}
$$


Since the inertial basis tensors are covariantly constant, we can write the field equations (15) as

$$
\square \Psi^{(a b)}=-16 \pi \mathcal{T}^{(a b)}
$$

where $\square$ is the simple scalar d'Alembertian. From the relations in Eqs. (64)-(70) and (771)-(77) we can then write

$$
e^{i k \Omega t} \square\left(e^{-i k \Omega t} \widetilde{\Psi}^{(a b)}\right)=-16 \pi \widetilde{\mathcal{T}}^{(a b)} .
$$

with $k=0 \pm 1, \pm 2$.

Lastly, for a helical scalar $f$ we have that

$$
e^{i k \Omega t} \square\left(e^{-i k \Omega t} f\right)=\square f+2 i k \Omega \partial_{t} f+k^{2} \Omega^{2} f .
$$

The time derivative $\partial_{t} f$ here uses the time coordinate of the inertial $t, r, \theta, \phi$ system and, for $f$ a (helically symmetric) function of the corotating coordinates $t, r, \theta, \varphi$, is equivalent to $-\Omega \partial_{\varphi} f$. The field equations for the metric perturbations then take the form

$$
\square \widetilde{\Psi}^{(a b)}-2 i k \Omega^{2} \partial_{\varphi} \widetilde{\Psi}+k^{2} \Omega^{2} \widetilde{\Psi}=-16 \pi \widetilde{\mathcal{T}}^{(a b)}
$$

where $\square$ is the scalar d'Alembertian. The explicit equations are

$$
\begin{aligned}
\square \widetilde{\Psi}^{(00)} & =-16 \pi \widetilde{\mathcal{T}}^{(00)} \\
\square \widetilde{\Psi}^{(n 0)} & =-16 \pi \widetilde{\mathcal{T}}^{(n 0)} \\
\square \widetilde{\Psi}^{(20)} & =-16 \pi \widetilde{\mathcal{T}}^{(20)} \\
\square \widetilde{\Psi}^{(n n)} & =-16 \pi \widetilde{\mathcal{T}}^{(n n)} \\
\square \widetilde{\Psi}^{(n 1)}-2 i \Omega^{2} \partial_{\varphi} \widetilde{\Psi}^{(n 1)}+\Omega^{2} \widetilde{\Psi}^{(n 1)} & =-16 \pi \widetilde{\mathcal{T}}^{(n 1)} \\
\square \widetilde{\Psi}^{(21)}-2 i \Omega^{2} \partial_{\varphi} \widetilde{\Psi}^{(21)}+\Omega^{2} \widetilde{\Psi}^{(21)} & =-16 \pi \widetilde{\mathcal{T}}^{(21)} \\
\square \widetilde{\Psi}^{(22)}-4 i \Omega^{2} \partial_{\varphi} \widetilde{\Psi}^{(22)}+4 \Omega^{2} \widetilde{\Psi}^{(22)} & =-16 \pi \widetilde{\mathcal{T}}^{(22)} .
\end{aligned}
$$

From Eqs. (29) - (34), and the prescriptions in Eqs. (56)- (62), and Eqs. (64)- (70), we have the inner boundary conditions, to be applied at small $\chi$ to represent the near field of the mass points at $\chi=0$,

$$
\begin{aligned}
& \widetilde{\Psi}^{(n n)}=4 m_{0} \frac{\gamma^{2}}{\sqrt{\widetilde{r}^{2}+\gamma^{2} v^{2} \widetilde{y}^{2}}} \\
& \widetilde{\Psi}^{(00)}=4 m_{0} \frac{\gamma^{2}}{\sqrt{\widetilde{r}^{2}+\gamma^{2} v^{2} \widetilde{y}^{2}}} \frac{v^{2}}{\sqrt{3}} \\
& \widetilde{\Psi}^{(20)}=4 m_{0} \frac{\gamma^{2}}{\sqrt{\widetilde{r}^{2}+\gamma^{2} v^{2} \widetilde{y}^{2}}}\left(-\frac{v^{2}}{\sqrt{6}}\right) \\
& V^{(n 1)}=4 m_{0} \frac{\gamma^{2}}{\sqrt{\widetilde{r}^{2}+\gamma^{2} v^{2} \widetilde{y}^{2}}} v \operatorname{sgn}[\cos \Theta] \\
& U^{(22)}=4 m_{0} \frac{\gamma^{2}}{\sqrt{\widetilde{r}^{2}+\gamma^{2} v^{2} \widetilde{y}^{2}}}\left(-\frac{v^{2}}{2}\right) \\
& \widetilde{\Psi}^{(n 0)}=U^{(21)}=V^{(21)}=U^{(n 1)}=V^{(22)}=0 .
\end{aligned}
$$

\section{Outer boundary conditions for helical scalars}

The quantities $\Psi^{(n n)}, \Psi^{(00)}, \Psi^{(n 0)}, \Psi^{(20)}$, are nonradiative multipoles that fall off as $1 / r$. The radiative parts of these fields satisfy a Sommerfeld condition

$$
\frac{\partial}{\partial r}[r \widetilde{\Psi}]= \pm \Omega \frac{\partial}{\partial \varphi}[r \widetilde{\Psi}]
$$


in which the upper and lower signs correspond respectively to outgoing and ingoing waves. In practice, special attention is not necessary for the nonradiatable multipoles of $\Psi^{(n n)}, \Psi^{(00)}, \Psi^{(n 0)}, \Psi^{(20)}$. The Sommerfeld conditions can be applied to the total field, without regard to multipole content.

Some care must be taken with the Sommerfeld conditions for $\widetilde{\Psi}^{(n 1)}, \widetilde{\Psi}^{(21)}$ and $\widetilde{\Psi}^{(22)}$. The Sommerfeld condition applies to the inertial projections of $\bar{h}_{\alpha \beta}$, and hence to $\Psi^{(n 1)}, \Psi^{(21)}$ and $\Psi^{(22)}$. With the relations, in Eqs. (64)- (70), between these quantities and the "helical scalars" $\widetilde{\Psi}^{(n 1)}, \widetilde{\Psi}^{(21)}, \widetilde{\Psi}^{(22)}$ used in computation, we arrive at the conditions

$$
\begin{gathered}
\frac{\partial}{\partial r}\left[r e^{i \varphi} \widetilde{\Psi}^{(n 1)}\right]= \pm \Omega \frac{\partial}{\partial \varphi}\left[r e^{i \varphi} \widetilde{\Psi}^{(n 1)}\right] \\
\frac{\partial}{\partial r}\left[r e^{i \varphi} \widetilde{\Psi}^{(21)}\right]= \pm \Omega \frac{\partial}{\partial \varphi}\left[r e^{i \varphi} \widetilde{\Psi}^{(21)}\right] \\
\frac{\partial}{\partial r}\left[r e^{2 i \varphi} \widetilde{\Psi}^{(22)}\right]= \pm \Omega \frac{\partial}{\partial \varphi}\left[r e^{2 i \varphi} \widetilde{\Psi}^{(22)}\right],
\end{gathered}
$$

or

$$
\begin{aligned}
& \frac{1}{r} \frac{\partial}{\partial r}\left(r U^{(a b)}\right)= \pm \Omega\left(-k V^{(a b)}+\frac{\partial U^{(a b)}}{\partial \varphi}\right) \\
& \frac{1}{r} \frac{\partial}{\partial r}\left(r V^{(a b)}\right)= \pm \Omega\left(k U^{(a b)}+\frac{\partial V^{(a b)}}{\partial \varphi}\right)
\end{aligned}
$$

in which $k=1$ for $(a b)=(n 1)$ or $(21)$, and $k=2$ for $(a b)=(22)$.

\section{Series solutions}

To develop series solutions of Eqs. (82) - [88) we start by using the expressions in Eqs. (18) - (20), for stress-energy components of the symmetric pair of particles, in the general expressions in Eqs. (71) - (77). The nonvanishing results are

$$
\begin{aligned}
\tilde{\mathcal{T}}^{(n n)} & =m_{0} \gamma \frac{\delta(r-a)}{a^{2}} \delta(\theta-\pi / 2)[\delta(\varphi)+\delta(\varphi-\pi)] \\
\tilde{\mathcal{T}}^{(n 1)}= & \pm i v \widetilde{\mathcal{T}}^{(n n)} \\
\tilde{\mathcal{T}}^{(20)}=-\frac{v^{2}}{\sqrt{6}} \tilde{\mathcal{T}}^{(n n)} & \widetilde{\mathcal{T}}^{(00)}=\frac{v^{2}}{\sqrt{3}} \widetilde{\mathcal{T}}^{(n n)} \\
\widetilde{\mathcal{T}}^{(22)}= & -\frac{v^{2}}{2} \widetilde{\mathcal{T}}^{(n n)} .
\end{aligned}
$$

As in Eq. (19), the upper sign in the expression for $\widetilde{T}^{(n 1)}$ indicates the particle at $\varphi=0$, the lower sign indicates the particle at $\varphi=\pi$.

With these expressions, Eqs. (82) - 888) take the explicit form

$$
\begin{gathered}
\square \widetilde{\Psi}^{(n n)}=-16 \pi m_{0} \frac{\delta(r-a)}{a^{2}} \delta(\cos \theta) \gamma[\delta(\varphi)+\delta(\varphi-\pi)] \\
\widetilde{\Psi}^{(n 0)}=0 \\
\square \widetilde{\Psi}^{(00)}=-16 \pi m_{0} \frac{\delta(r-a)}{a^{2}} \delta(\cos \theta) \frac{v^{2} \gamma}{\sqrt{3}}[\delta(\varphi)+\delta(\varphi-\pi)] \\
\square \widetilde{\Psi}^{(20)}=+16 \pi m_{0} \frac{\delta(r-a)}{a^{2}} \delta(\cos \theta) \frac{v^{2} \gamma}{\sqrt{6}}[\delta(\varphi)+\delta(\varphi-\pi)]
\end{gathered}
$$




$$
\begin{gathered}
\square \widetilde{\Psi}^{(n 1)}-2 i \Omega^{2} \partial_{\varphi} \widetilde{\Psi}^{(n 1)}+\Omega^{2} \widetilde{\Psi}^{(n 1)}=-i 16 \pi m_{0} \frac{\delta(r-a)}{a^{2}} \delta(\cos \theta) v \gamma[\delta(\varphi)-\delta(\varphi-\pi)] \\
\widetilde{\Psi}^{(21)}=0 \\
\square \widetilde{\Psi}^{(22)}-4 i \Omega^{2} \partial_{\varphi} \widetilde{\Psi}^{(22)}+4 \Omega^{2} \widetilde{\Psi}^{(22)}=+16 \pi m_{0} \frac{\delta(r-a)}{a^{2}} \delta(\cos \theta) \frac{v^{2} \gamma}{2}[\delta(\varphi)+\delta(\varphi-\pi)]
\end{gathered}
$$

where

$$
\square \widetilde{\Psi}=\frac{1}{r^{2}} \frac{\partial}{\partial r}\left(r^{2} \frac{\partial \widetilde{\Psi}}{\partial r}\right)+\frac{1}{r^{2} \sin \theta} \frac{\partial}{\partial \theta}\left(\sin \theta \frac{\partial \widetilde{\Psi}}{\partial \theta}\right)+\left(\frac{1}{r^{2} \sin ^{2} \theta}-\Omega^{2}\right) \frac{\partial^{2} \widetilde{\Psi}}{\partial \varphi^{2}}
$$

For $\widetilde{\Psi}^{(n n)}, \widetilde{\Psi}^{(20)}, \widetilde{\Psi}^{(00)}$ the equations all have the form

$$
\square \widetilde{\Psi}=K \frac{\delta(r-a)}{a^{2}} \delta(\cos \theta)[\delta(\varphi)+\delta(\varphi-\pi)]
$$

in which the value of $K$ can be read from Eqs. (105) - (108). The outoging solutions are constructed in the usual manner from the spherical Bessel and Hankel functions:

$$
\Psi=-2 i \Omega K \sum_{\ell, m \text { even }} m j_{\ell}\left(m \Omega r_{<}\right) h_{\ell}^{(1)}\left(m \Omega r_{>}\right) Y_{\ell m}^{*}(\pi / 2,0) Y_{\ell m}(\theta, \varphi)
$$

or

$$
\begin{aligned}
\Psi & =-2 K \sum_{\ell} \frac{1}{2 \ell+1} Y_{\ell 0}^{*}(\pi / 2,0) Y_{\ell 0}(\theta, 0) \frac{r_{<}^{\ell}}{r_{>}^{\ell+1}} \\
& +4 K \Omega \sum_{\ell} \sum_{m=2,4,6} m Y_{\ell m}^{*}(\pi / 2,0) Y_{\ell m}(\theta, 0) j_{\ell}\left(m \Omega r_{<}\right) \operatorname{Im}\left\{h_{\ell}^{(1)}\left(m \Omega r_{>}\right) e^{i m \varphi}\right\}
\end{aligned}
$$

By expansion in spherical harmonics, then by the usual Green function construction, the outgoing solution for $\widetilde{\Psi}^{(n 1)}$ is found to be

$$
\begin{aligned}
& \widetilde{\Psi}^{(n 1)}=U^{(n 1)}+i V^{(n 1)}=-32 \pi m_{0} v \gamma \sum_{\ell}\left[Y_{\ell,-1}^{*}(\pi / 2,0) Y_{\ell,-1}(\theta, 0)\left(\frac{-i e^{-i \varphi}}{2 \ell+1}\right) \frac{r_{<}^{\ell}}{r_{>}^{\ell+1}}\right. \\
& \left.+\Omega \sum_{m=\text { odd }, \neq-1}(m+1) Y_{\ell, m}^{*}(\pi / 2,0) Y_{\ell, m}(\theta, 0) j_{\ell}\left((m+1) \Omega r_{<}\right) h_{\ell}^{(1)}\left((m+1) \Omega r_{>}\right) e^{i m \varphi}\right]
\end{aligned}
$$

or

$$
\begin{gathered}
U^{(n 1)}=-32 \pi m_{0} v \gamma \sum_{\ell \text { odd }}\left[\frac{-\sin \varphi}{(2 \ell+1)} \frac{r_{<}^{\ell}}{r_{>}^{\ell+1}} Y_{\ell-1}^{*}(\pi / 2,0) Y_{\ell-1}(\theta, 0)\right. \\
+\Omega \sum_{m=1,3,5, \cdots}(m+1) Y_{\ell m}^{*}(\pi / 2,0) Y_{\ell m}(\theta, 0) j_{\ell}\left((m+1) \Omega r_{<}\right)\left[\cos m \varphi j_{\ell}\left((m+1) \Omega r_{>}\right)-\sin m \varphi n_{\ell}\left((m+1) \Omega r_{>}\right)\right]
\end{gathered}
$$

$\left.-\Omega \sum_{m=1,3,5, \cdots}(m+1) Y_{\ell,-m-2}^{*}(\pi / 2,0) Y_{\ell,-m-2}(\theta, 0) j_{\ell}\left((m+1) \Omega r_{<}\right)\left[\cos ((m+2) \varphi) j_{\ell}\left((m+1) \Omega r_{>}\right)-\sin ((m+2) \varphi) n_{\ell}\left((m+1) \Omega r_{>}\right)\right]\right]$ 
and

$$
\begin{gathered}
V^{(n 1)}=-32 \pi m_{0} v \gamma \sum_{\ell \text { odd }}\left[\frac{-\cos \varphi}{(2 \ell+1)} \frac{r_{<}^{\ell}}{r_{>}^{\ell+1}} Y_{\ell,-1}^{*}(\pi / 2,0) Y_{\ell,-1}(\theta, 0)\right. \\
+\Omega \sum_{m=1,3,5 \cdots}(m+1) Y_{\ell m}^{*}(\pi / 2,0) Y_{\ell m}(\theta, 0) j_{\ell}\left((m+1) \Omega r_{<}\right)\left[\cos m \varphi n_{\ell}\left((m+1) \Omega r_{>}\right)+\sin m \varphi j_{\ell}\left((m+1) \Omega r_{>}\right)\right] \\
\left.+\Omega \sum_{m=1,3,5 \cdots}(m+1) Y_{\ell,-m-2}^{*}(\pi / 2,0) Y_{\ell,-m-2}(\theta, 0) j_{\ell}\left((m+1) \Omega r_{<}\right)\left[\cos ((m+2) \varphi) n_{\ell}\left((m+1) \Omega r_{>}\right)+\sin ((m+2) \varphi) j_{\ell}\left((m+1) \Omega r_{>}\right)\right]\right]
\end{gathered}
$$

Similarly, the outgoing solution for $\widetilde{\Psi}^{(22)}$ is found to be

$$
\begin{gathered}
\widetilde{\Psi}^{(22)}=U^{(22)}+i V^{(22)}=16 \pi m_{0} v^{2} \gamma \sum_{\ell=\mathrm{even}}\left[Y_{\ell,-2}^{*}(\pi / 2,0) Y_{\ell,-2}(\theta, 0)\left(\frac{-e^{-2 i \varphi}}{2 \ell+1}\right) \frac{r_{<}^{\ell}}{r_{>}^{\ell+1}}\right. \\
\left.-i \Omega \sum_{m=\text { even }, \neq-2}(m+2) Y_{\ell, m}^{*}(\pi / 2,0) Y_{\ell, m}(\theta, 0) j_{\ell}\left((m+2) \Omega r_{<}\right) h_{\ell}^{(1)}\left((m+2) \Omega r_{>}\right) e^{i m \varphi}\right]
\end{gathered}
$$

or

$$
\begin{gathered}
U^{(22)}=16 \pi m_{0} v^{2} \gamma \sum_{\ell \text { even }}\left[\frac{-\cos 2 \varphi}{(2 \ell+1)} \frac{r_{<}^{\ell}}{r_{>}^{\ell+1}} Y_{\ell,-2}^{*}(\pi / 2,0) Y_{\ell,-2}(\theta, 0)\right. \\
+\Omega \sum_{m=0,2,4 \cdots}(m+2) Y_{\ell m}^{*}(\pi / 2,0) Y_{\ell m}(\theta, 0) j_{\ell}\left((m+2) \Omega r_{<}\right)\left[\cos m \varphi n_{\ell}\left((m+2) \Omega r_{>}\right)+\sin m \varphi j_{\ell}\left((m+2) \Omega r_{>}\right)\right] \\
\left.+\Omega \sum_{m=0,2,4 \cdots}(m+2) Y_{\ell,-m-4}^{*}(\pi / 2,0) Y_{\ell,-m-4}(\theta, 0) j_{\ell}\left((m+2) \Omega r_{<}\right)\left[\cos ((m+4) \varphi) n_{\ell}\left((m+2) \Omega r_{>}\right)+\sin ((m+4) \varphi) j_{\ell}\left((m+2) \Omega r_{>}\right)\right]\right]
\end{gathered}
$$

and

$$
\begin{gathered}
V^{(22)}=16 \pi m_{0} v^{2} \gamma \sum_{\ell \text { even }}\left[\frac{\sin 2 \varphi}{(2 \ell+1)} \frac{r_{<}^{\ell}}{r_{>}^{\ell+1}} Y_{\ell,-2}^{*}(\pi / 2,0) Y_{\ell,-2}(\theta, 0)\right. \\
+\sum_{m=0,2,4 \cdots}(m+2) Y_{\ell m}^{*}(\pi / 2,0) Y_{\ell m}(\theta, 0) j_{\ell}\left((m+2) \Omega r_{<}\right)\left[\sin m \varphi n_{\ell}\left((m+2) \Omega r_{>}\right)-\cos m \varphi j_{\ell}\left((m+2) \Omega r_{>}\right)\right]
\end{gathered}
$$

$$
\underset{m=0,2,4 \cdots}{\left.-\Omega \sum_{\ell-m-4}(m / 2,0) Y_{\ell,-m-4}(\theta, 0) j_{\ell}\left((m+2) \Omega r_{<}\right)\left[\sin ((m+4) \varphi) n_{\ell}\left((m+2) \Omega r_{>}\right)-\cos ((m+4) \varphi) j_{\ell}\left((m+2) \Omega r_{>}\right)\right]\right] .}
$$

The expressions for $U^{(n 1)}, V^{(n 1)}, U^{(22)}$, and $V^{(22)}$ have been given as sums only over nonnegative values of $m$ through the use of the relationship $j_{\ell}(-x) h_{\ell}^{(1)}(-x)=j_{\ell}(x)\left(h_{\ell}^{(1)}(x)\right)^{*}$. 


\section{LINEARIZED GRAVITY IN ADAPTED COORDINATES}

\section{A. Field equations in adapted coordinates}

The field equations to be used for computation are the source-free forms of Eqs. (82) - 887). The first four of these equations are

$$
\square \widetilde{\Psi}^{(a b)}=0
$$

for $(a b)=(00),(n 0),(20)$, and $(n 0)$. In real form the last three of these equations are

$$
\begin{aligned}
& \square U^{(a b)}+2 k \Omega^{2} \partial_{\varphi} V^{(a b)}+k^{2} \Omega^{2} U^{(a b)}=0 \\
& \square V^{(a b)}-2 k \Omega^{2} \partial_{\varphi} U^{(a b)}+k^{2} \Omega^{2} V^{(a b)}=0
\end{aligned}
$$

with $k=1$ for $(a b)=(n 1)$, or $(21)$, and $k=2$ for $(a b)=(22)$.

The form of the scalar d'Alembertian in adapted coordinates is given in Eq. (8) of Ref. [12], as

$$
\begin{gathered}
\square \Psi=A_{\chi \chi} \frac{\partial^{2} \Psi}{\partial \chi^{2}}+A_{\Theta \Theta} \frac{\partial^{2} \Psi}{\partial \Theta^{2}}+A_{\Phi \Phi} \frac{\partial^{2} \Psi}{\partial \Phi^{2}}+2 A_{\chi \Theta} \frac{\partial^{2} \Psi}{\partial \chi \partial \Theta}+2 A_{\chi \Phi} \frac{\partial^{2} \Psi}{\partial \chi \partial \Phi}+2 A_{\Theta \Phi} \frac{\partial^{2} \Psi}{\partial \Theta \partial \Phi} \\
+B_{\chi} \frac{\partial \Psi}{\partial \chi}+B_{\Theta} \frac{\partial \Psi}{\partial \Theta}+B_{\Phi} \frac{\partial \Psi}{\partial \Phi}
\end{gathered}
$$

and the adapted-coordinate form of $\partial_{\varphi}$ in Eq. (27) of Ref. 12],

$$
\frac{\partial}{\partial \varphi}=\left(\Gamma^{\Theta} \frac{\partial \Psi}{\partial \Theta}+\Gamma^{\Phi} \frac{\partial \Psi}{\partial \Phi}+\Gamma^{\chi} \frac{\partial \Psi}{\partial \chi}\right)
$$

Here the $A, B$ and $\Gamma$ coefficients are known, real functions of $\chi, \Theta, \Phi$ that are given explicitly in Appendix A of Ref. 12] and are repeated in the appendix of the present paper.

\section{B. Inner boundary conditions in adapted coordinates}

To express the inner boundary conditions in terms of the adapted coordinates, we approximate

$$
\begin{aligned}
\widetilde{r}^{2}+\gamma^{2} v^{2} \widetilde{y}^{2}=(\widetilde{Z}-a)^{2}+\widetilde{X}^{2}+\widetilde{Y}^{2}+\gamma^{2} v^{2} \widetilde{X}^{2}= \\
{\left[1+\gamma^{2} v^{2} \sin ^{2} 2 \Theta \cos ^{2} \Phi\right] \frac{\chi^{4}}{4 a^{2}}+\mathcal{O}\left(\chi^{6} / a^{4}\right), }
\end{aligned}
$$

and we write Eqs. (89)-(94), for the $\chi \rightarrow 0$ limits of the the fields, as

$$
\begin{aligned}
\widetilde{\Psi}^{(n n)} & =4 m_{0} \frac{2 a \gamma^{2}}{\chi^{2} \sqrt{1+\gamma^{2} v^{2} \sin ^{2} 2 \Theta \cos ^{2} \Phi}} \\
\widetilde{\Psi}^{(00)} & =4 m_{0} \frac{2 a \gamma^{2}}{\chi^{2} \sqrt{1+\gamma^{2} v^{2} \sin ^{2} 2 \Theta \cos ^{2} \Phi}} \frac{v^{2}}{\sqrt{3}} \\
\widetilde{\Psi}^{(20)} & =4 m_{0} \frac{2 a \gamma^{2}}{\chi^{2} \sqrt{1+\gamma^{2} v^{2} \sin ^{2} 2 \Theta \cos ^{2} \Phi}}\left(-\frac{v^{2}}{\sqrt{6}}\right) \\
V^{(n 1)} & =4 m_{0} \frac{2 a \gamma^{2}}{\chi^{2} \sqrt{1+\gamma^{2} v^{2} \sin ^{2} 2 \Theta \cos ^{2} \Phi}} v \operatorname{sgn}[\cos \Theta] \\
U^{(22)} & =4 m_{0} \frac{2 a \gamma^{2}}{\chi^{2} \sqrt{1+\gamma^{2} v^{2} \sin ^{2} 2 \Theta \cos ^{2} \Phi}}\left(-\frac{v^{2}}{2}\right) \\
\widetilde{\Psi}^{(n 0)} & =U^{(21)}=V^{(21)}=U^{(n 1)}=V^{(22)}=0 .
\end{aligned}
$$




\section{Outer boundary conditions in adapted coordinates}

The outer boundary conditions in adapted coordinates follow from Eqs. (95) - 987 with $r$ replaced by $\chi$ and $\partial_{\varphi}$ replaced by the expressions in Eq. (126). For the fields $\widetilde{\Psi}^{(n n)}, \widetilde{\Psi}^{(00)}, \widetilde{\Psi}^{(n 0)}$ and $\widetilde{\Psi}^{(20)}$, the conditions are just those used for the scalar field in Ref. [12]. For the complex fields, the conditions are a modification of Eqs. (99) and (100), and are the tensorial equivalent of the adapted-coordinate outer boundary condition (11) used for scalar fields; aside from corrections of order $(\chi / a)^{2}$ these results are:

$$
\begin{aligned}
& \frac{1}{\chi} \frac{\partial}{\partial \chi}\left(\chi U^{(a b)}\right)= \pm \Omega\left(-k V^{(a b)}+\Gamma^{\Theta} \frac{\partial U^{(a b)}}{\partial \Theta}+\Gamma^{\Phi} \frac{\partial U^{(a b)}}{\partial \Phi}+\Gamma^{\chi} \frac{\partial U^{(a b)}}{\partial \chi}\right) \\
& \frac{1}{\chi} \frac{\partial}{\partial \chi}\left(\chi V^{(a b)}\right)= \pm \Omega\left(k U^{(a b)}+\Gamma^{\Theta} \frac{\partial V^{(a b)}}{\partial \Theta}+\Gamma^{\Phi} \frac{\partial V^{(a b)}}{\partial \Phi}+\Gamma^{\chi} \frac{\partial V^{(a b)}}{\partial \chi}\right)
\end{aligned}
$$

where

$$
k=1 \text { for }(a b)=(n 1),(n 0),(21) \quad k=2 \text { for }(a b)=(22) .
$$

\section{NUMERICAL RESULTS}

The field equations in adapted coordinates for $\widetilde{\Psi}^{(n n)}, \widetilde{\Psi}^{(00)}, \widetilde{\Psi}^{(n 0)}$ and $\widetilde{\Psi}^{(20)}$ are defined by the homogeneous wave equation (122) and by the form of the d'Alembertian in Eq. (125) with the coefficients in Appendix $\mathrm{A}$ These equations are subject to the inner boundary conditions in Eqs. (128) - 130) applied at some smallest value $\chi_{\min }$ of $\chi$. The equations must satisfy the outgoing or ingoing outer boundary conditions Eq. (11) applied at a largest computational value $\chi_{\max }$ of $\chi$. In the linear theory each of the scalar-like fields $\widetilde{\Psi}^{(n n)}, \widetilde{\Psi}^{(00)}, \widetilde{\Psi}^{(00)}, \widetilde{\Psi}^{(20)}$ is completely decoupled from every other fields, both in the field equations and in the boundary conditions. Furthermore, the field equations, and boundary conditions have precisely the same forms as those for the scalar field problem. The computational problem, therefore, is precisely that of Ref. [12] where it was shown that the computed solution agrees accurately with the numerically evaluated series solution in Eq. (115).

In the computation of the complex fields $\widetilde{\Psi}^{(n 1)}$ and $\widetilde{\Psi}^{(22)}$, features arise that are different from those in Ref. 12]. Here the field equations (123) and (124), and the outer boundary conditions (11), couple the real and imaginary parts. [This would apply also to the complex field $\widetilde{\Psi}^{(21)}$, but due to the inner boundary condition $\widetilde{\Psi}^{(21)}=0$ in Eq. (133), the field $\widetilde{\Psi}^{(21)}$ must be be identically zero in linearized theory.]

The series solutions for $U^{(n 1)}$ and $V^{(n 1)}$ are given in Eqs. (117) - 118) and those for $U^{(22)}$ and $V^{(22)}$ in Eqs. (120) - (121). Figures 4 and 5 give a comparison, for two different source velocities, between these series solutions and the solutions of the eigenspectral method: adapted coordinates and multipole filtering based on the modified multipoles appropriate to the discrete angular operator.

A feature that stands out in the figures is the disagreement at small $\chi$ between the series and eigenspectral solutions for $V^{(n 1)}$ and for $U^{(22)}$. The failing here is in the convergence of the series solutions in Eqs. (118) and (120). The $V^{(n 1)}$ and $U^{(22)}$ fields diverge at $\chi \rightarrow 0$, hence these series converge very slowly at small $\chi$. Numerical experiments summing very large numbers of terms and evalutating them with arbitrary precision arithmetic confirm that the series solutions for $V^{(n 1)}$ and $U^{(22)}$ in Figs. 4 and 5 have large errors. The series for $U^{(n 1)}$ and $V^{(22)}$, on the other hand, are convergent at $\chi=0$ and show good agreement with the small- $\chi$ form of the eigenspectral solutions.

The series solutions are highly accurate for $\chi>1$, so the differences between the series solutions and the eigenspectral solutions for large $\chi$ are an indication of the limitations of the eigenspectral method. Those differences are larger for $\Omega>0.4$ than for $\Omega>0.3$. Computations (not presented here) for $\Omega>0.5$ show significantly larger error. The origin of these errors is the relatively coarse computational grid used, and the limited number of multipoles used in the multipole filtering. (This is equivalent in the computation to the coarseness of the angular grid.) The error is simply that due to truncation error, and is expected. As $\Omega$ is increased the gradients of the fields increase and truncation error becomes more important. These limitations are imposed by the fact that the computation was done on a $2 \mathrm{~GB}$ RAM workstation. Greater accuracy, and hence higher velocity, would be possible on larger machines,

To test whether our numerical techniques might be sufficient for the next steps in our program, we introduced a simple nonlinearity into the equations of Sec. IV

$$
\square \bar{h}^{n n}=\square \widetilde{\Psi}^{(n n)}=\kappa \frac{S}{H^{2}+a^{2} S}
$$



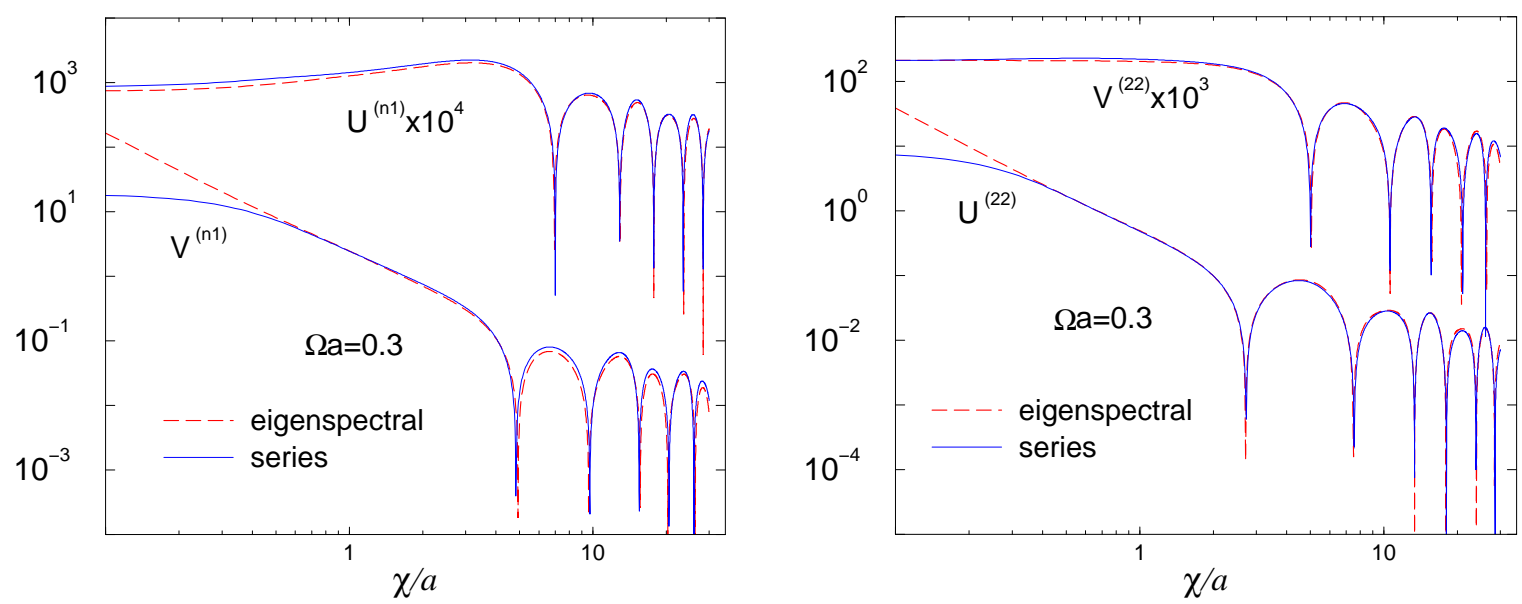

FIG. 4: A comparison, for $a \Omega=0.3$, of outgoing linearized gravitational fields computed by series summation (solid curve), and by the solution of the coupled partial differential equations of the "eigenspectral" method of the PSW approach (dashed curve). The fields are shown along a line (the $\Theta=0$ line) outward through the source. For these computations, the grid in $\chi$, $\Theta, \Phi$ was $1500,16,32$, respectively. The entire angular space, $0 \leq \Theta \leq \pi, 0 \leq \Phi \leq 2 \pi$, was used. The inner boundary was at $\chi_{\min }=0.1 a$ and the outgoing boundary condition was imposed at $\chi_{\max }=30 a$. The multipole filtering included all modes up through the octupole, approximately $\ell=3$. (The actual discrete eigenvalues differ slightly from integer values.) See the text for a discussion of the numerical limitations of the series summation.
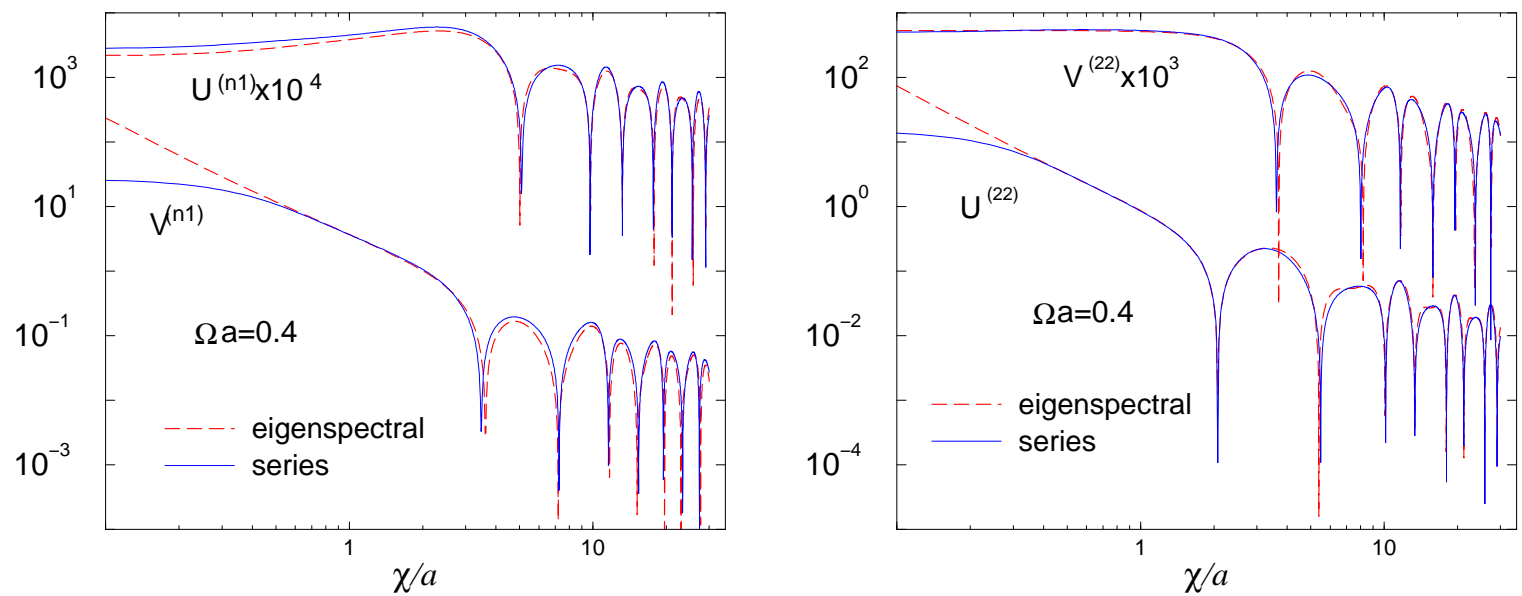

FIG. 5: A comparison, for $a \Omega=0.4$, of outgoing linearized gravitational fields computed by series summation (solid curve), and by the solution of the coupled partial differential equations of the "eigenspectral" method of the PSW approach (dashed curve). The details of the computations are the same as those for Fig. 4

with $S$ defined by

$$
S=\eta^{\lambda \sigma} \bar{h}_{, \lambda}^{n \alpha} \bar{h}_{, \sigma}^{n \beta} \eta_{\alpha \beta}
$$

where $\bar{h}^{n \alpha}=(\mathbf{n} \cdot \overline{\mathbf{h}})^{\alpha}$.

With the notation of Eqs. (54) this can be written as

$$
S=-\eta^{\lambda \sigma} \Psi_{, \lambda}^{(n n)} \Psi_{, \sigma}^{(n n)}+\eta^{\lambda \sigma} \Psi_{, \lambda}^{(n 0)} \Psi_{, \sigma}^{(n 0)}+\eta^{\lambda \sigma} \Psi_{, \lambda}^{(n 1)}\left(\Psi^{(n 1)}\right)_{, \sigma}^{*} .
$$

Since $\Psi^{(n n)}$ and $\Psi^{(n 0)}$ are "helical scalars," i.e., functions only of corotating coordinates, for $(a b)=(n n)$ or $(n 0)$, we have

$$
\begin{aligned}
& \eta^{\lambda \sigma} \Psi_{, \lambda}^{(a b)} \Psi_{, \sigma}^{(a b)}=\eta^{\lambda \sigma} \widetilde{\Psi}_{, \lambda}^{(a b)} \widetilde{\Psi}_{, \sigma}^{(a b)}= \\
& \quad-\Omega^{2} \widetilde{\Psi}_{, \varphi}^{(a b)} \widetilde{\Psi}_{, \varphi}^{(a b)}+(\nabla \chi \cdot \nabla \chi) \widetilde{\Psi}_{, \chi}^{(a b)} \widetilde{\Psi}_{, \chi}^{(a b)}+(\nabla \Theta \cdot \nabla \Theta) \widetilde{\Psi}_{, \Theta}^{(a b)} \widetilde{\Psi}_{, \Theta}^{(a b)}+(\nabla \Phi \cdot \nabla \Phi) \widetilde{\Psi}_{, \Phi}^{(a b)} \widetilde{\Psi}_{, \Phi}^{(a b)}
\end{aligned}
$$


in which

$$
\begin{aligned}
\vec{\nabla} \chi \cdot \vec{\nabla} \chi & =\frac{Q}{\chi^{2}} \\
\vec{\nabla} \Theta \cdot \vec{\nabla} \Theta & =\frac{Q}{\chi^{4}} \\
\vec{\nabla} \Phi \cdot \vec{\nabla} \Phi & =2 \frac{Q+a^{2}+\chi^{2} \cos (2 \Theta)}{\chi^{4} \sin ^{2}(2 \Theta)} \\
Q & \equiv \sqrt{a^{4}+2 a^{2} \chi^{2} \cos (2 \Theta)+\chi^{4}} .
\end{aligned}
$$

For $\Psi^{(n 1)}$ we have $\left(\Psi^{(n 1)}=e^{-i \Omega t} \widetilde{\Psi}^{(n 1)}\right.$ which results in

$$
\begin{gathered}
\eta^{\lambda \sigma} \Psi_{, \lambda}^{(n 1)}\left(\Psi_{, \sigma}^{(n 1)}\right)^{*}=-\Omega^{2} \widetilde{\Psi}^{(n 1)}\left(\widetilde{\Psi}^{(n 1)}\right)^{*}-i \Omega^{2}\left[\widetilde{\Psi}^{(n 1)}\left(\widetilde{\Psi}_{, \varphi}^{(n 1)}\right)^{*}-\widetilde{\Psi}_{, \varphi}^{(n 1)}\left(\widetilde{\Psi}^{(n 1)}\right)^{*}\right]-\Omega^{2} \widetilde{\Psi}_{, \varphi}^{(n 1)}\left(\widetilde{\Psi}_{, \varphi}^{(n 1)}\right)^{*} \\
+(\nabla \chi \cdot \nabla \chi) \widetilde{\Psi}_{, \chi}^{(n 1)}\left(\widetilde{\Psi}_{, \chi}^{(n 1)}\right)^{*}+(\nabla \Theta \cdot \nabla \Theta) \widetilde{\Psi}_{, \Theta}^{(n 1)}\left(\widetilde{\Psi}_{, \Theta}^{(n 1)}\right)^{*}+(\nabla \Phi \cdot \nabla \Phi) \widetilde{\Psi}_{, \Phi}^{(n 1)}\left(\widetilde{\Psi}_{, \Phi}^{(n 1)}\right)^{*} \\
=-\Omega^{2}\left[\left(U^{(n 1)}\right)^{2}+\left(V^{(n 1)}\right)^{2}\right]+2 \Omega^{2}\left[V^{(n 1)} U_{, \varphi}^{(n 1)}-U^{(n 1)} V_{, \varphi}^{(n 1)}\right]-\Omega^{2}\left[\left(U_{, \varphi}^{(n 1)}\right)^{2}+\left(V_{, \varphi}^{(n 1)}\right)^{2}\right] \\
+(\nabla \chi \cdot \nabla \chi)\left[\left(U_{, \chi}^{(n 1)}\right)^{2}+\left(V_{, \chi}^{(n 1)}\right)^{2}\right]+(\nabla \Theta \cdot \nabla \Theta)\left[\left(U_{, \Theta}^{(n 1)}\right)^{2}+\left(V_{, \Theta}^{(n 1)}\right)^{2}\right]+(\nabla \Phi \cdot \nabla \Phi)\left[\left(U_{, \Phi}^{(n 1)}\right)^{2}+\left(V_{, \Phi}^{(n 1)}\right)^{2}\right] .
\end{gathered}
$$

In the modified "theory" represented by Eq. (137) the equations that determine $\widetilde{\Psi}^{(n 0)}$ and $\widetilde{\Psi}^{(n 1)}$ remain unchanged, so these fields are found with linear equations. The nonlinear occurrence of $\widetilde{\Psi}^{(n n)}$ in $S$, however, means that $\widetilde{\Psi}^{(n n)}$ solves a nonlinear equation.

The solution to this nonlinear problem requires the iterative techniques that have previously been used for nonlinear scalar models in Ref. 12]. Results from the applications of these iterative methods to the modified theory are displayed in Fig. [6 for various values of the nonlinearity parameter $\kappa$. For all values of $\kappa$ the inner boundary conditions are taken to be those of the linear problem and are imposed at $\chi_{\min }=0.1$; for all models outgoing boundary conditions are imposed at $\chi_{\max }=30$. A comparison, in that figure, with the $\kappa=0$ linear solution demonstrates that the crosscoupling of fields and nonlinearity has a strong effect on the solution to the toy model, changing the amplitude of the $\widetilde{\Psi}^{(n n)}$ waves by an order of magnitude. The purpose of this nonlinear toy computation is not to extract physics, but simply to suggest that the iteration techniques previously developed will be adequate at least for a range of nonlinear models.

\section{SUMMARY AND DISCUSSION}

This paper has laid out the infrastructure for describing helically symmetric linearized gravity, and more generally for describing helically symmetric second-rank tensors in a flat background. The fields have been written in terms of "helical scalars," i.e., functions only of corotating coordinates (equivalently, labels on the curves of the Killing congruence). The paper has, furthermore, shown how to formulate computational problems in linearized general relativity in terms of these helical scalars. The field equations, sources, and inner and outer boundary conditions have been written in this format in Sec. III]

A welcome feature of the formulation in Sec. III is the extent of the separation of "components" of the field. In the starting point, the Lorentz-gauge field equations $\bar{h}_{\mu \nu, \alpha}^{, \alpha}=-16 \pi T_{\mu \nu}$, a separate equation is satisfied by each component $\bar{h}_{\mu \nu}$ with respect to an inertial Minkowski basis. That attractive feature cannot be taken over directly to the helically symmetric problem since the components with respect to the inertial basis are not helical scalars. It turns out, however, that the formulation of the linear problem in helical scalars leads to four real fields $\tilde{\Psi}^{(n n)}, \tilde{\Psi}^{(n 0)}$, $\tilde{\Psi}^{(00)}$ and $\tilde{\Psi}^{(20)}$, and three complex fields $\tilde{\Psi}^{(n 1)}, \tilde{\Psi}^{(21)}$ and $\tilde{\Psi}^{(22)}$, and that these four real and three complex fields are not mixed by the field equations or the inner or outer boundary conditions. The only mixing in the problem is between the real and imaginary parts of the complex fields.

Since the three corotating coordinates are general, this paper has also presented the explicit formulation of the computational problem in the adapted coordinates that were found in Ref. [12] to be extremely useful. The numerical 


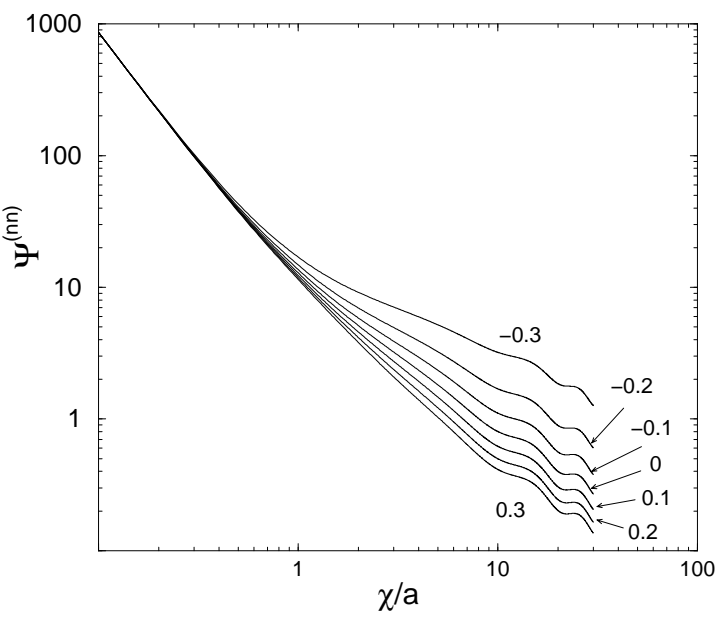

FIG. 6: The field $\widetilde{\Psi}^{(n n)}(\chi)$ for the toy nonlinear model described in the text. Curves are marked with the value of the nonlinearity parameter $\kappa$; the parameter $H$ was set to unity and the models were run on a $1500 \times 16 \times 32$ grid for the full $\chi, \Theta, \Phi$ space (i.e., no symmetries were used), with $\chi_{\min } / a=0.1$, and $\chi_{\max } / a=30$. In the multipole filtering, modes through octupole were included for all fields. Comparison with the $\kappa=0$ linear solution shows the importance of nonlinear effects.

results in Sec. $\mathbb{\nabla}$ of the present paper demonstrate that the numerical challenges presented by linearized gravity are the same as those for the linear scalar model in Ref. [12]. Indeed, the numerical problem for the four real fields is exactly the same as that for the linear scalar field. For the complex fields, the new features are minor modifications of the boundary conditions and mixing of the real part and of the imaginary part of each of the complex fields. These new features do not appear to present any new numerical difficulty, and in fact no difficulty was found. Furthermore, a trial with a toy nonlinearity suggests that there are also no new problems in dealing with nonlinear terms, except those of complexity.

Although the present paper deals almost exclusively with linearized gravity, the infrastructure developed here is more widely applicable. Our next step in the PSW program is to solve the post-Minkowskian problem for the orbiting point masses. The equations to be solved in this method have the same operator $\bar{h}_{\mu \nu, \alpha}{ }_{\alpha}^{\alpha}$ on the left hand side, but have "sources" quadratic in $\bar{h}_{\mu \nu, \alpha}$ on the right. (There is also a second derivative on the right multiplied by an undifferentiated $\bar{h}_{\mu \nu}$; this term can be treated, like the others, as a perturbation, or it can be moved to the left to modify the principal part.) In the usual spirit of a post-Minkowski approximation, we could solve first for the firstorder fields and treat them as known sources. Alternatively, in a numerical approach, we could treat the equations as a given nonlinear problem. Either way, the formalism developed in the present paper goes over directly to the post-Minkowskian problem. Again, the fields can be described with four real and three complex helical scalars, and the equations and boundary conditions follow from the simple relations between the helical scalars and their inertial equivalents, i.e., relations like those in Eqs. (64) - (700).

The full Einstein equations can also be viewed as a higher order extension of the post-Minkowski equations. In principle, the only change from the post-Minkowski problem is the inclusion of terms of all orders on the nonlinear right-hand side of the field equations. Again the formalism developed in the present paper should suffice for the description of the problem, and should be convenient. In particular, helical scalars of the background Minkowski space will be helical scalars of the full metric. In practice, new problems will arise. One is the question of the relativistic Kepler's law: what is the appropriate relationship relating the source strength (encoded in inner boundary conditions), the coordinate separation of the sources, and the parameter $\Omega$ ? The post-Minkowskian approximation, for which the answer is known, will help clarify how this is to be handled in full general relativity. A more subtle question is whether it is justified to use a formalism based on weak-field structures to describe strong gravitational fields. For highly curved spacetimes do Minkowksi-like coordinates exist with which we can use the formalism of the present paper? Possibly relevant to this is the fact that in our computations we can impose inner boundary condistions at some distance from the sources, so that the effects of strong fields can be somewhat controlled.

In any case, the relative simplicity of the description presented here, along with the absence of any new computational difficulties (that is, difficulties not present in the scalar problem) is a reason for optimism that the next steps can be taken reasonably quickly. 


\section{ACKNOWLEDGMENT}

We gratefully acknowledge the support of NSF grant PHY0244605 and NASA grant ATP03-0001-0027, to UTB. We thank Kip Thorne, Lee Lindblom, Mark Scheel and the Caltech numerical relativity group, and John Friedman.

\section{APPENDIX A: COEFFICIENTS FOR ADAPTED COORDINATES}

The adapted-coordinate coefficient $A, B$, and $\Gamma$ are listed here. Derivations are provided in Ref. [12].

As in Ref. [12] the coefficients needed for the wave operator in Eq. (125) are written in the form:

$$
\begin{aligned}
A_{\chi \chi} & =\frac{Q}{\chi^{2}}-\Omega^{2} \bar{A}_{\chi \chi} \\
A_{\Theta \Theta} & =\frac{Q}{\chi^{4}}-\Omega^{2} \bar{A}_{\Theta \Theta} \\
A_{\Phi \Phi} & =2 \frac{Q+a^{2}+\chi^{2} \cos (2 \Theta)}{\chi^{4} \sin ^{2}(2 \Theta)}-\Omega^{2} \bar{A}_{\Phi \Phi} \\
A_{\chi \Theta} & =-\Omega^{2} \bar{A}_{\chi \Theta} \\
A_{\chi \Phi} & =-\Omega^{2} \bar{A}_{\chi \Phi} \\
A_{\Theta \Phi} & =-\Omega^{2} \bar{A}_{\Theta \Phi} \\
B_{\chi} & =\frac{a^{2}+2 Q}{\chi^{3}}-\Omega^{2} \bar{B}_{\chi} \\
B_{\Theta} & =\frac{\sqrt{Q+a^{2}+\chi^{2} \cos (2 \Theta)}}{\sqrt{Q-a^{2}-\chi^{2} \cos (2 \Theta)}} \frac{\left(Q-a^{2}\right)}{\chi^{4}}-\Omega^{2} \bar{B}_{\Theta} \\
B_{\Phi} & =-\Omega^{2} \bar{B}_{\Phi},
\end{aligned}
$$

where $Q$ is given in Eq. (144).

The expressions multiplied by $\Omega^{2}$ are:

$$
\begin{gathered}
\bar{A}_{\chi \chi}=\frac{a^{4} \sin ^{2}(2 \Theta) \cos ^{2} \Phi}{\chi^{2}} \\
\bar{A}_{\Theta \Theta}=\frac{\cos ^{2} \Phi\left[\chi^{2}+a^{2} \cos (2 \Theta)\right]^{2}}{\chi^{4}} \\
\bar{A}_{\Phi \Phi}=\sin ^{2} \Phi \frac{Q+a^{2}+\chi^{2} \cos (2 \Theta)}{Q-a^{2}-\chi^{2} \cos (2 \Theta)} \\
\bar{A}_{\chi \Theta}=\frac{a^{2}\left[\chi^{2}+a^{2} \cos (2 \Theta)\right] \sin (2 \Theta) \cos { }^{2} \Phi}{\chi^{3}} \\
\bar{A}_{\chi \Phi}=-\frac{a^{2}\left[Q+a^{2}+\chi^{2} \cos (2 \Theta)\right] \sin \Phi \cos \Phi}{\chi^{3}} \\
\bar{A}_{\Theta \Phi}=-\frac{\sin (\Phi) \cos (\Phi)\left[a^{2}+\chi^{2} \cos (2 \Theta)+Q\right]\left[\chi^{2}+a^{2} \cos (2 \Theta)\right]}{\chi^{4} \sin (2 \Theta)} \\
\bar{B}_{\chi}=\frac{a^{2}\left[\cos ^{2}(\Phi)\left\{3 a^{2} \cos ^{2}(2 \Theta)-Q-2 a^{2}+\chi^{2} \cos (2 \Theta)\right\}+Q+a^{2}+\chi^{2} \cos (2 \Theta)\right]}{\chi^{3}}
\end{gathered}
$$




$$
\begin{gathered}
\bar{B}_{\Phi}=\frac{\left(3 Q+a^{2}+\chi^{2} \cos 2 \Theta\right) \sin (\Phi) \cos (\Phi)}{Q-a^{2}-\chi^{2} \cos 2 \Theta} \\
\bar{B}_{\Theta}=\frac{\sqrt{Q+a^{2}+\chi^{2} \cos (2 \Theta)}}{\chi^{6} \sqrt{Q-a^{2}-\chi^{2} \cos (2 \Theta)}}\left(c \cos ^{2} \Phi+d\right)
\end{gathered}
$$

where

$$
\begin{gathered}
c \equiv a^{2} \chi^{4} \cos (2 \Theta)+2 a^{4} \chi^{2}+4 a^{6} \cos (2 \Theta)+4 a^{4} \chi^{2}(\cos (2 \Theta))^{2}-4 a^{4} Q \cos (2 \Theta)-2 a^{2} Q \chi^{2}-\chi^{6} \\
d \equiv \chi^{4}\left(a^{2} \cos (2 \Theta)+\chi^{2}\right) .
\end{gathered}
$$

The coefficients needed in Eq. (126) a to express the Sommerfeld boundary condition in adapted coordinates are:

$$
\begin{gathered}
\Gamma^{\chi}=\left(\widetilde{Z} \frac{\partial \chi}{\partial \widetilde{X}}-\widetilde{X} \frac{\partial \chi}{\partial \widetilde{Z}}\right)=\frac{a^{2} \cos \Phi \sin (2 \Theta)}{\chi} \\
\Gamma^{\Theta}=\left(\widetilde{Z} \frac{\partial \Theta}{\partial \widetilde{X}}-\widetilde{X} \frac{\partial \Theta}{\partial \widetilde{Z}}\right)=\frac{\cos \Phi\left(a^{2} \cos (2 \Theta)+\chi^{2}\right)}{\chi^{2}} \\
\Gamma^{\Phi}=\left(\widetilde{Z} \frac{\partial \Phi}{\partial \widetilde{X}}-\widetilde{X} \frac{\partial \Phi}{\partial \widetilde{Z}}\right)=-\frac{\chi^{2} \sin \Phi \sin (2 \Theta)}{-a^{2}-\chi^{2} \cos 2 \Theta+Q}
\end{gathered}
$$

Note that there were errors in the expressions given for the $\Gamma$ s in Ref. [12].

[1] M. Campanelli, C. O. Lousto, P. Marronetti, and Y. Zlochower, preprint gr-qc/0511048. M. Campanelli, C. O. Lousto, and Y. Zlochower, preprint gr-qc/0601091

[2] J. G. Baker, J. Centrella, D.-I. Choi, M. Koppitz, and J. van Meter, preprint gr-qc/0505100

[3] J. Baker, M. Campanelli, C. O. Lousto, and R. Takahashi, Phys. Rev. D 65, 124012 (2002).

[4] L. Blanchet, Living Rev. Relativity 5, (2002), 3. [Online article]: $\quad$ cited Dec. 25, 2000 http://www.livingreviews.org/lrr-2002-3

[5] J. K. Blackburn and S. Detweiler, Phys. Rev. D 46, 2318 (1992).

[6] S. Detweiler, Phys. Rev. D 50, 4929 (1994).

[7] J. T. Whelan, W. Krivan, and R. H. Price, Class. Quant. Grav. 17, 4895 (2000).

[8] J. T. Whelan, C. Beetle, W. Landry, and R. H. Price, Class. Quant. Grav. 19, 1285 (2002).

[9] R. H. Price, Class. Quant. Grav. 21, S281 (2004).

[10] Z. Andrade et al., Phys. Rev. D 70, 064001 (2004).

[11] M. Shibata, J. L. Friedman, K. Uryu, and M. Shibata Phys. Rev. D 65, 064035 (2002). Erratum-ibid. D 70129904 (2004).

[12] B. Bromley, R. Owen and R. H. Price Phys. Rev. D 71, 104017 (2005).

[13] C. W. Misner, K. S. Thorne and J. A. Wheeler, Gravitation (W. H. Freeman, San Francisco, 1973).

[14] C. G. Torre, J. Math. Phys., 44 6223-6232 (2003).

[15] F. J. Zerilli, Phys. Rev. D 2, 2141 (1970), Eq. (1).

[16] K. S. Thorne Rev. Mod. Phys. 52, 299 (1980), Sec. IIE.

[17] J. Mathews, J. Soc. Ind. Appl. Math. 10, 768 (1962).

[18] T. Regge and J. A. Wheeler, Phys. Rev. 108, 1063 (1957).

[19] V. D. Sandberg, J. Math. Phys 19, 2441 (1978).

[20] J. N. Goldberg, A. J. MacFarlane, E. T Newman, F. Rohrlich and E. C. G. Sudarshan, J. Math. Phys 8, 2155 (1967). 